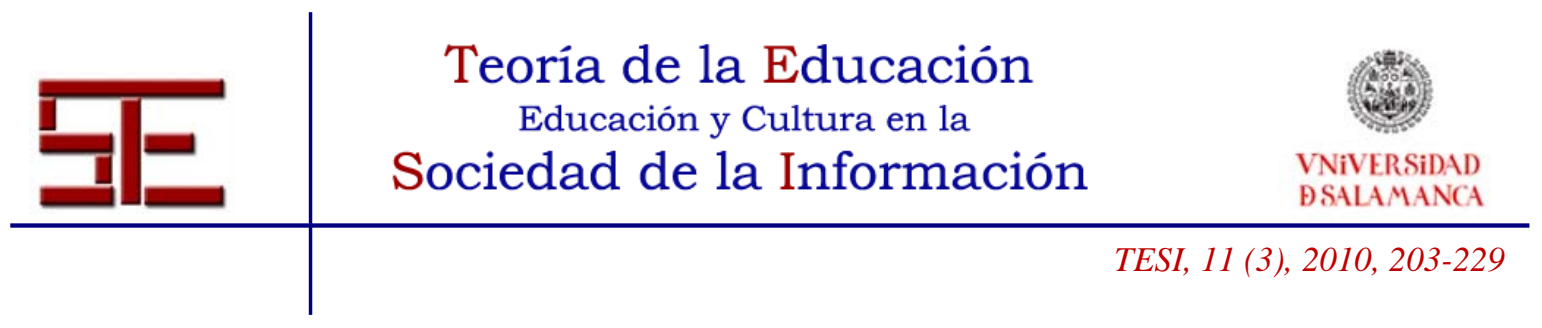

\title{
ENSEÑAR Y APRENDER CON TECNOLOGÍAS: UN MODELO TEÓRICO PARA LAS BUENAS PRÁCTICAS CON TIC ${ }^{1}$
}

\section{Resumen:}

Este artículo tiene como objetivo definir un marco teórico explicativo de la integración de las Tecnologías de la Información y la Comunicación (TIC) en el sistema educativo, desde la perspectiva de la formación del profesorado. Esta formación inicial y continua de los docentes se caracteriza por una tendencia hacia la "esencialización" de la tecnología y la generación de usuarios que no suelen reflexionar sobre los usos educativos de la propia tecnología en contextos flexibles y variables. Nuestra investigación sobre la integración de las TIC en las aulas nos ha permitido observar la falta de conexión entre los usos personales y profesionales de los profesores de estas herramientas tecnológicas, así como la necesidad de que su formación se oriente hacia el desarrollo de conocimientos y habilidades para examinar, de manera crítica, las implicaciones educativas de estos nuevos medios didácticos. Partimos de la propuesta de Koehler \& Mishra (2005, 2006, 2007 y 2008) denominada TPCK (Technological Pedagogical Content Knowledge) que se fundamenta en el constructo PCK de Shulman (1987) al que se añade el concepto de “Tecnología” (T), a los de "Pedagogía” (P) y "Contenido Curricular" (C). Las conexiones e interacciones dinámicas entre estos tres componentes esenciales dan lugar a diferentes componentes que han de ser considerados en la comprensión de los procesos de integración de las TIC en los centros educativos. Las Buenas $\mathrm{P}$ rácticas educativas con TIC son acciones complejas y multidimensionales que exigen (1) comprender la representación y formulación de conceptos y procedimientos para su comprensión a través de las TIC; (2) desarrollar estrategias didácticas constructivistas que usen las TIC para la enseñanza de contenidos curriculares; (3) conocer las dificultades en el aprendizaje de conceptos y de qué forma las TIC pueden ayudar a superarlas y (4) conocer el conocimiento previo de los

\footnotetext{
${ }^{1}$ La investigación que ha servido de base para este artículo, “Análisis de las políticas educativas para la integración y uso de las TIC en el sistema educativo de Extremadura y sus efectos en la innovación didáctica.”, ha sido financiada por el Ministerio de Ciencia e Innovación. Dirección General de Programas y Transferencia del Conocimiento. Plan Nacional de I+D+I 2006-2009. Ref. SEJ200612435-C05-05/EDUC. Llevada a cabo por el grupo de investigación reconocido “Nodo Educativo”, del cual es director Jesús Valverde Berrocoso.
}

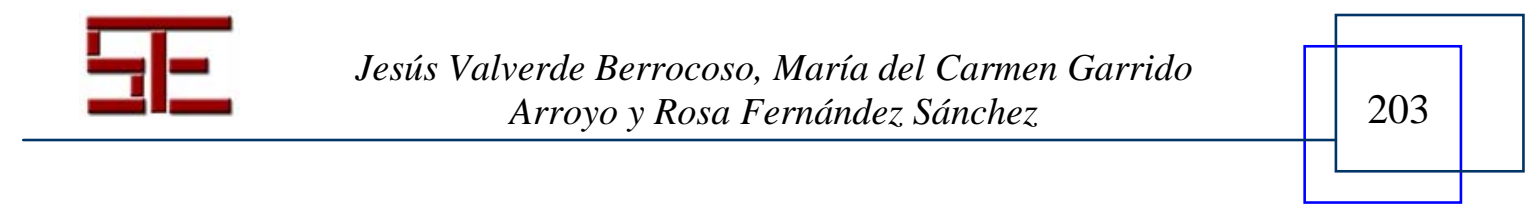




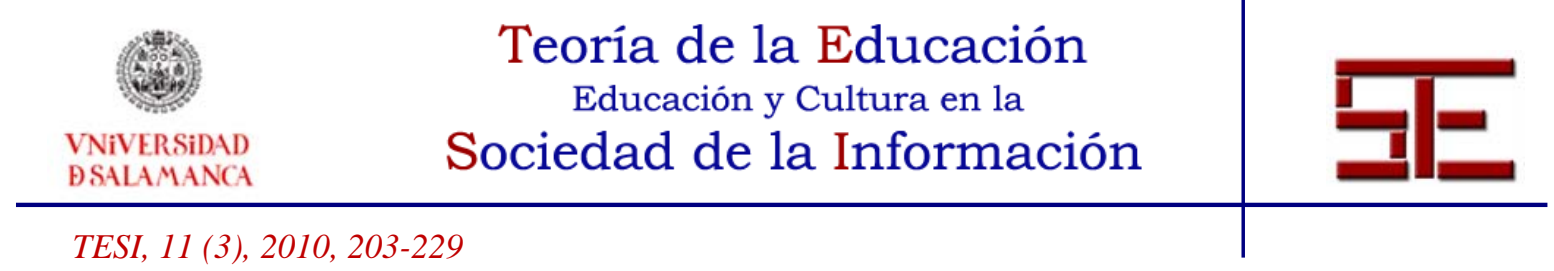

alumnos, así como la epistemología del contenido curricular para comprender cómo las TIC pueden ser utilizadas para construir sobre el conocimiento preexistente y desarrollar nuevas epistemologías. Estos conocimientos claramente van más allá del que posee aisladamente un experto en un contenido curricular (profesor de una disciplina), un experto en TIC (ingeniero) o un pedagogo experto (tecnólogo educativo), por lo que la formación del profesorado exige un replanteamiento de los enfoques y las prácticas actuales.

Palabras clave:

Tecnología educativa, formación del profesorado, Tecnologías de la Información y la Comunicación (TIC), modelo teórico, integración de las TIC. 


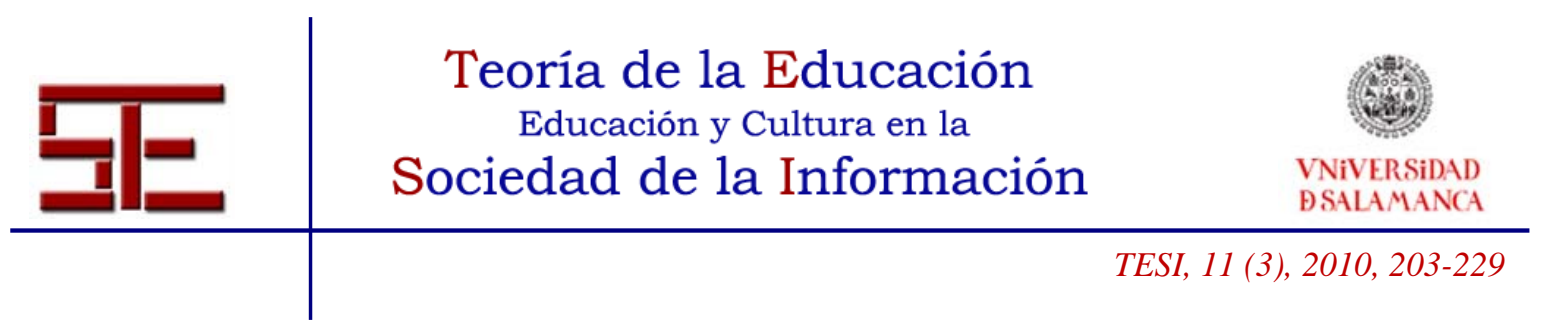

\title{
TEACHING AND LEARNING WITH TECHNOLOGY: A THEORETICAL MODEL FOR GOOD EDUCATIONAL PRACTICES WITHICT
}

\begin{abstract}
:
This article aims to define a theoretical explanatory framework for the integration of information technologies and communication technologies (ICT) in education from the perspective of teacher education. The initial and continuing training of teachers is characterized by a tendency towards "essentialisation" of technology and generation of users who do not usually think about educational uses of technology in their own contexts. Our research on the integration of ICT in the classroom has allowed us to observe the lack of connection between the personal and professional use of teachers of these technological tools, as well as the need for training is geared towards developing skills and knowledge to examine, in a critical manner, the educational implications of these new teaching aids. This article is based on the proposed Koehler \& Mishra (2005, 2006, 2007 and 2008) called TPCK (Technological Pedagogical Content Knowledge) which is based on the construct of PCK Shulman (1987) to which is added the concept of "Technology" (T) to those of "Pedagogy" (P) and "Curriculum Content" (C). Connections and dynamic interactions between these three key components leading to different components to be considered in understanding the processes of integration of ICT in schools. Good educational practices with ICT are multidimensional and complex actions that require (1) understand the representation and formulation of concepts and procedures for their understanding through ICT, (2) develop constructivist teaching strategies that use ICT for teaching content curriculum, (3) know the difficulties in learning concepts and how ICT can help overcome them, and (4) knowing the students' prior knowledge and the epistemology of the curriculum to understand how ICT can be used to build on pre-existing knowledge and develop new epistemologies. These skills clearly go beyond the isolation that has an expert in a curriculum (teacher of a discipline), an expert in IT (engineer), or an expert teacher (educational technologist), so the teacher education requires a rethinking approaches and current practices.
\end{abstract}

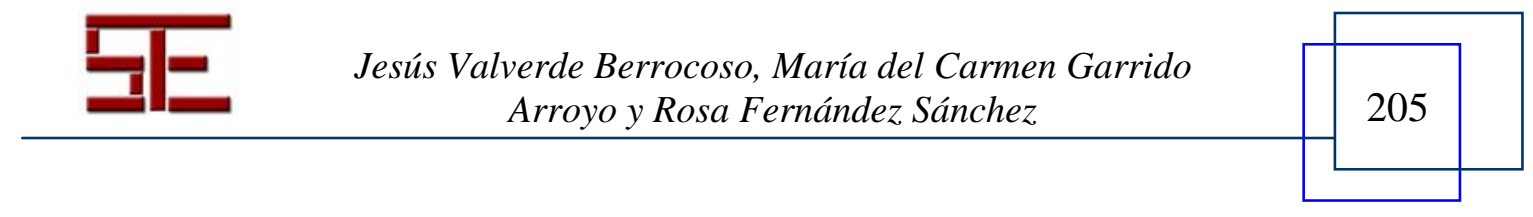




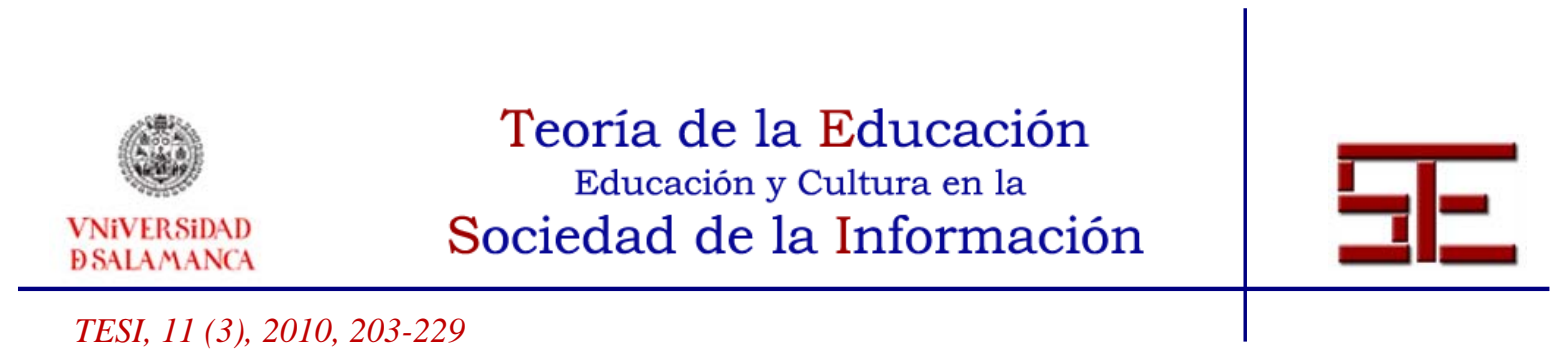

Key words:

Educational technology, teacher education, Technologies of Information and Communication (ICT), theoretical model, ICT intregration.

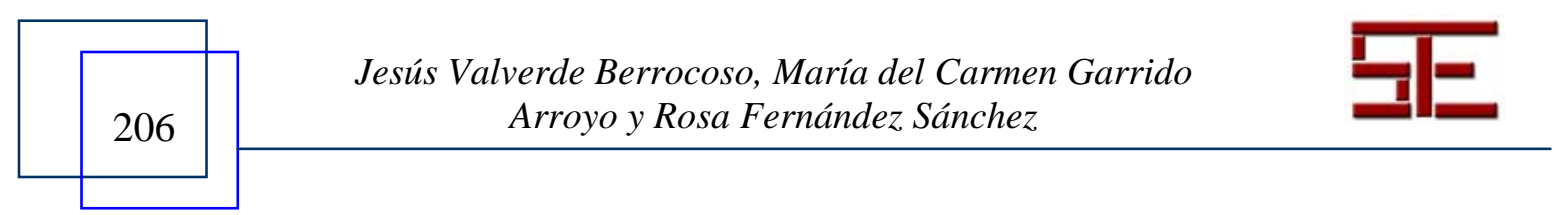




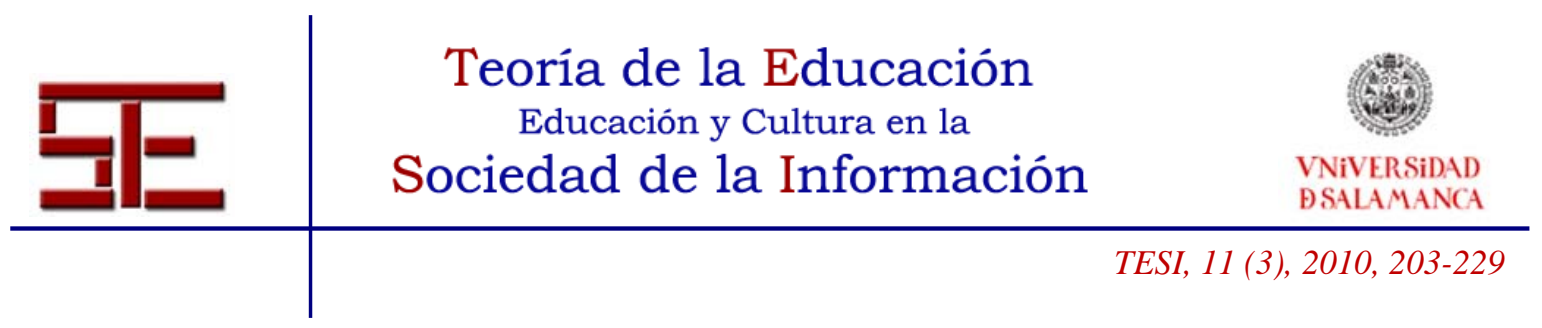

\section{ENSEÑAR Y APRENDER CON TECNOLOGÍAS: UN MODELO TEÓRICO PARA LAS BUENAS PRÁCTICAS CON TIC}

Fecha de recepción: 10/02/2009; fecha de aceptación: 03/12/2009; fecha de publicación: 28/02/10

Jesús Valverde Berrocoso

jevabe@unex.es

Universidad de Extremadura.

María del Carmen Garrido Arroyo

cargarri@unex.es

Universidad de Extremadura.

Rosa Fernández Sánchez

rofersan@unex.es

Universidad de Extremadura.

\section{1.- TECNOLOGÍA EDUCATIVA: COMPLEJIDAD Y NECESIDAD DE UN MODELO TEÓRICO}

La actividad profesional de los profesores tiene lugar en un contexto caracterizado por un elevado grado de complejidad y por un gran dinamismo, que les obliga a integrar conocimientos diversos que tienen que ver con el contenido curricular que enseñan, con la naturaleza de los procesos cognitivos y volitivos de sus alumnos y, en la actualidad, cada vez con mayor relevancia, con el conocimiento sobre el uso de tecnologías para el aprendizaje. La Pedagogía y la Didáctica son ejemplos paradigmáticos de disciplinas poco estructuradas, puesto que su objeto de conocimiento opera con un alto nivel de variabilidad y con una densa interconexión de conocimientos teórico-prácticos. Los procesos actuales de integración de las TIC en las aulas hacen aún más complejo este campo al introducirse nuevos ámbitos de conocimientos que el profesor debe dominar para desarrollar buenas prácticas educativas con tecnologías.

Gibson (1977) introdujo el término affordance (que aquí traducimos por «posibilidad») para referirse a las propiedades psicológicas percibidas y reales de cualquier objeto,

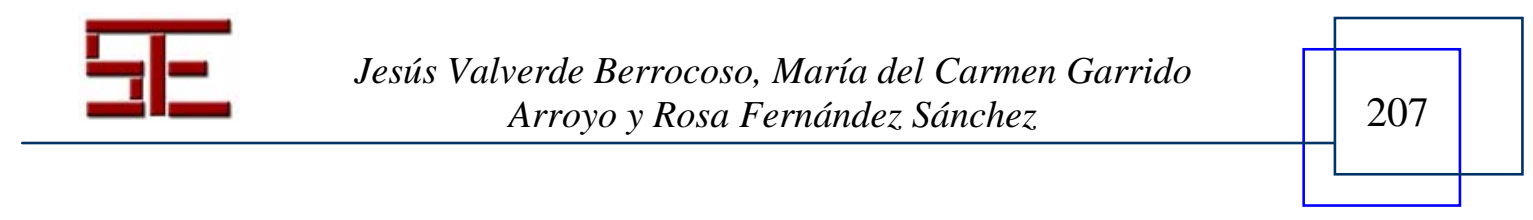




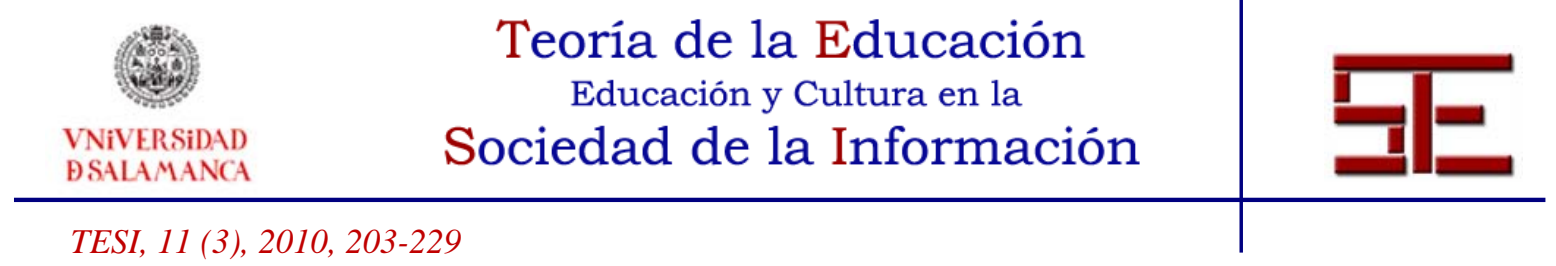

como medio de explicar cómo los individuos interactúan con los objetos del mundo. Pero es preciso diferenciar entre posibilidades y limitaciones inherentes a la tecnología de aquellas que son impuestas desde fuera por el usuario. Por lo general, interpretamos las TIC con nuestros propios prejuicios y predilecciones que tienen que ver con las formas de uso y aplicación que consideramos «apropiadas» o «inapropiadas». Las TIC tienen posibilidades y limitaciones como medios didácticos. No son, en ningún caso, herramientas neutrales. Precisamente los científicos cognitivos utilizan la expresión «fijación funcional» (funtional fixedness) para describir la forma en que las ideas que sostenemos sobre la función de un objeto pueden inhibir nuestra capacidad para usar el objeto para una función diferente. Los usos creativos de las TIC exigen ir más allá de esta «fijación funcional» de modo que podamos, de modo innovador, definir nuevos propósitos para las herramientas existentes y orientarlas hacia una finalidad educativa.

No se puede afirmar que la integración de las tecnologías sea un fenónemo novedoso en la educación formal. De hecho, la invención de la escritura y, más tarde, de la imprenta y su utilización en las aulas, fueron fuente de controversias y resistencias educativas, como ahora ocurre con las TIC. Podríamos aducir diferentes razones por las que la introducción de las TIC en el proceso de enseñanza-aprendizaje añade aún más complejidad al fenómeno educativo. Por una parte, encontramos a grupos sociales y organismos institucionales que, por diversos motivos, no apoyan suficientemente los esfuerzos de los profesores para integrar las tecnologías en su actividad docente. Por otro lado, es aún muy frecuente encontrar a profesores que no poseen la formación adecuada para llevar a cabo esta difícil integración en su actividad diaria. A todas estas dificultades se añade la diversidad de contextos de enseñanza-aprendizaje que nos muestran cómo no existe una «única vía» para llevar a cabo el proceso de integración de las TIC en las aulas. Incluso si nos movemos en un nivel más «micro» y analizamos tecnologías específicas para contenidos curriculares determinados, nos encontramos con un gran número de posibilidades tecnológicas para transformar las representaciones tradicionales del conocimiento escolar en modos de expresión más ricos y adaptados a los lenguajes audiovisuales e interactivos que dominan nuestra cultura, junto con un importante número de limitaciones sobre las funciones reales y prácticas que pueden cumplir en el aula.

Si examinamos las tecnologías educativas tradicionales observaremos que se caracterizan por su especificidad (un lápiz es para escribir o un microscopio es para visualizar objetos pequeños); su estabilidad (bolígrafos, microscopios o pizarras no cambian mucho con el tiempo); y la transparencia de su función (el funcionamiento interno de un lápiz es bastante simple y está relacionado directamente con su función). Su uso continuado y habitual las han convertido en tecnologías «transparentes» por ser herramientas docentes comunes, hasta el punto de dejar de ser conceptualizadas como «tecnologías». En contraste con las tecnologías tradicionales, las tecnologías digitales

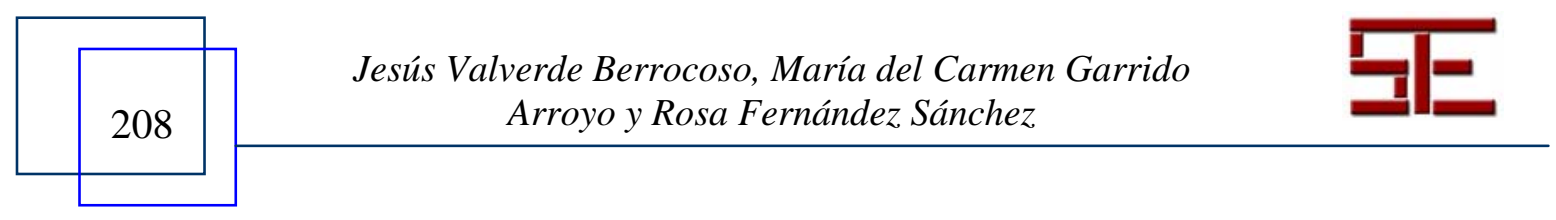




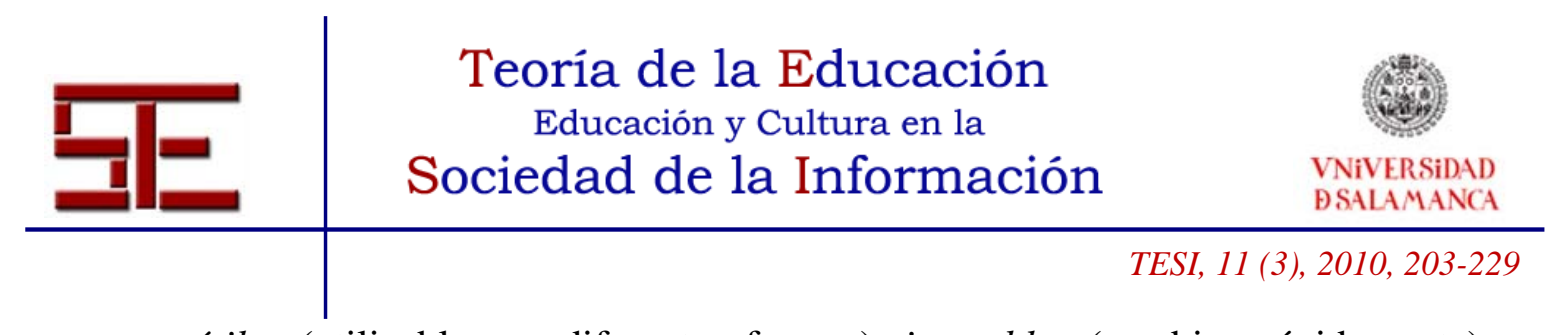

son versátiles (utilizables en diferentes formas), inestables (cambian rápidamente) y opacas (su funcionamiento interno está oculto al usuario).

La versatilidad de las tecnologías digitales hace que, por ejemplo, el ordenador sea una herramienta única para almacenar, distribuir y ayudar a manipular una variedad de sistemas de símbolos. Puede simular el funcionamiento de cualquier otro medio, constituyéndose en un meta-medio, con posibilidades para la representación y expresión como nunca antes han existido. Esta naturaleza versátil también significa que las tecnologías digitales hacen cosas muy diferentes según quién y para qué las utiliza. Es la razón por la cual el ordenador tiene aplicación prácticamente en cualquier campo de actividad humana. Sin embargo, su versatilidad también convierte al ordenador en una herramienta compleja en su uso y difícil en su aprendizaje. No ha de sorprender que su introducción en las aulas complique la actividad del profesor.

La inestabilidad de las TIC se manifiesta de dos formas. Por un lado, no existe un conocimiento estable y duradero para el aprendizaje de estas tecnologías. Su obsolescencia se manifiesta en ritmos de cambio muy acelerados que son difíciles de asumir por muchos usuarios. Se necesita estar continuamente al día de las demandas novedosas de estas tecnologías, es decir, ser un aprendiz continuo. Por otra parte, las tecnologías digitales no suelen tener un comportamiento fiable debido a su continua modificación y mejora. El software es un producto nunca acabado, siempre por pulir, susceptible de ser alterado para cumplir nuevas funciones. Esto afecta a su calidad y obliga al profesorado a moverse en el terreno de la ambigüedad, la frustración y el cambio. Para algunos profesores esto es difícilmente asumible y admisible dentro de un aula.

Por otra parte, el funcionamiento interno de la mayoría de las tecnologías digitales es opaco para quienes las utilizan. Esta cualidad hace que la interacción con el ordenador sea simbólica y, con frecuencia, arbitraria. El hecho de que la mayoría del software esté diseñado para contextos no educativos contribuye aún más a esta opacidad. Adaptar software de propósito general del entorno laboral (p ej. las hojas de cálculo) a la práctica escolar requiere trabajar a través de esta opacidad para reconfigurar y modificar sus propósitos iniciales a las necesidades educativas (Koehler \& Mishra, 2008).

La preparación de los profesores en los usos educativos de la tecnología es un componente clave en todos los planes de reforma educativa y factor clave en el desarrollo de Buenas Prácticas educativas con TIC (Area, 2004; Casanova, 2007; Correa y Blanco, 2004; De Pablos y Jiménez, 2007; Raposo et al., 2006; Valverde et al., 1999; Valverde, 2002). Aunque las administraciones educativas han dedicado en los últimos años un importante esfuerzo en la formación tecnológica del profesorado en ejercicio, lo cierto es que en la actualidad aún son muchos los profesores que no se

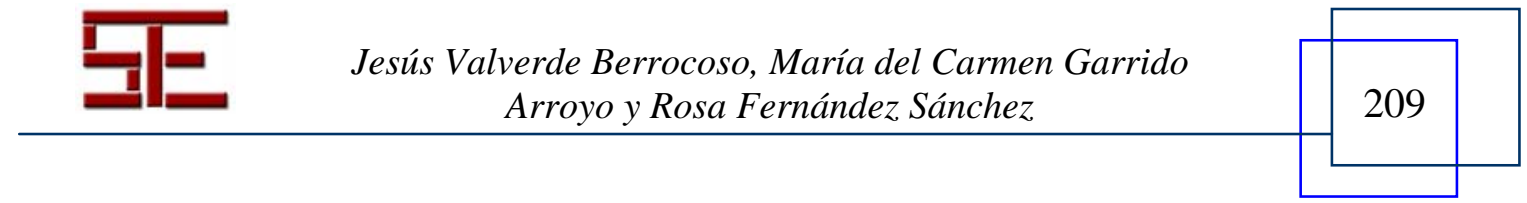




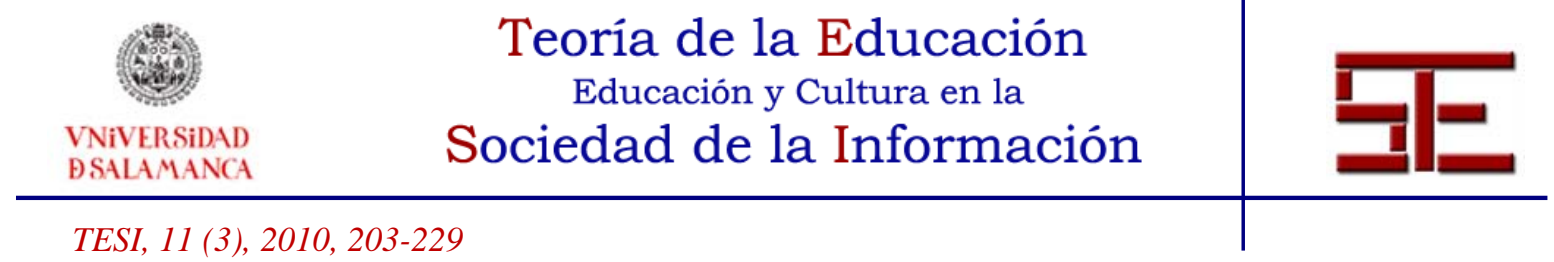

consideran competentes para abordar la integración de las TIC en sus prácticas docentes y que, en consecuencia, no han descubierto la relevancia de estos nuevos medios para el aprendizaje. Por consiguiente, los programas fallan en la preparación adecuada del profesorado ya que no establecen conexiones pedagógicas entre las posibilidades de las tecnologías y la enseñanza de un particular contenido curricular.

Además de las dificultades de compatibilizar su horario de trabajo con la formación en cursos y del sesgo excesivamente técnico que suelen adoptar los planes de formación en tecnología educativa, la adopción de las TIC en las aulas depende de que los usos educativos de las TIC sean compatibles con sus concepciones pedagógicas previas (Ertmer, 2005). La investigación sugiere que una innovación es menos probable que se adopte si se desvía demasiado de los valores previos, las creencias pedagógicas y las prácticas habituales del profesorado (Zhao, Pugh, Sheldon \& Byers, 2002). Recientemente, se están incorporando constructos que subrayan los elementos emocionales implicados en la innovación educativa con TIC (De Pablos, 2008). En la actualidad el asunto relevante no es si los profesores deben integrar la tecnología en sus prácticas docentes ya existentes, sino cómo usar la tecnología para transformar su enseñanza con tecnología y crear nuevas oportunidades para el aprendizaje (Angeli \& Valanides, 2009).

Para alcanzar buenas prácticas educativas con TIC, es necesario superar la falsa dicotomía entre pedagogía y tecnología. Por lo común, se consideran que ambos dominios están reglados por diferentes expertos que tienen muy poco que compartir. Según Koehler \& Mishra (2008), profesores y técnicos viven en mundos diferentes y, a menudo, poseen imágenes distorsionadas de cada uno de ellos. Por un lado, los técnicos ven a los profesores como luditas, conservadores, resistentes al cambio e inconscientes al poder transformador de las tecnologías. Y, por otro, los profesores tienden a ver a los técnicos como entusiastas superficiales, desconocedores de la educación y de las teorías del aprendizaje e inconscientes de las realidades del aula y las escuelas.

Entre los conocimientos que los profesores tienen que manejar para integrar las TIC en sus buenas prácticas, están las observaciones y el análisis de los diferentes contextos de aplicación en lo que cada uno enseña. No existe una solución única para resolver la introducción de las tecnologías en el currículum. Se exige el esfuerzo de rediseñar continuamente el uso de los medios para adaptarse a realidades cambiantes. Además, en el entorno escolar han de convivir los «nativos digitales» (primera generación de estudiantes que han vivido y crecido completamente alrededor de las tecnologías digitales) con los «inmigrantes digitales» (profesores que han «migrado» a estas tecnologías en edades más tardías) (Prensky, 2001, 2006).

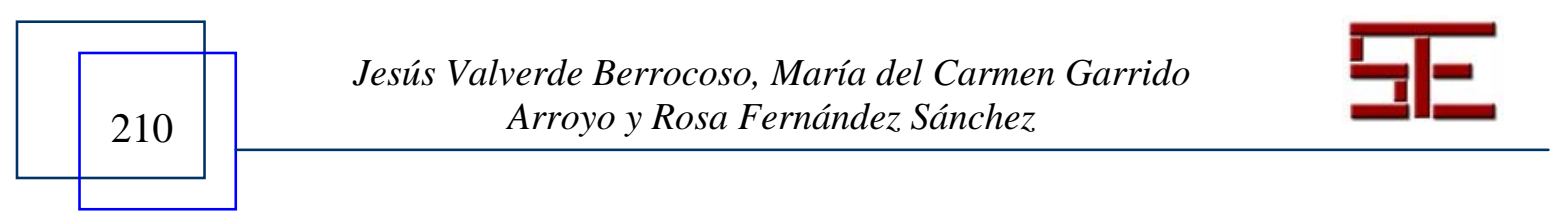




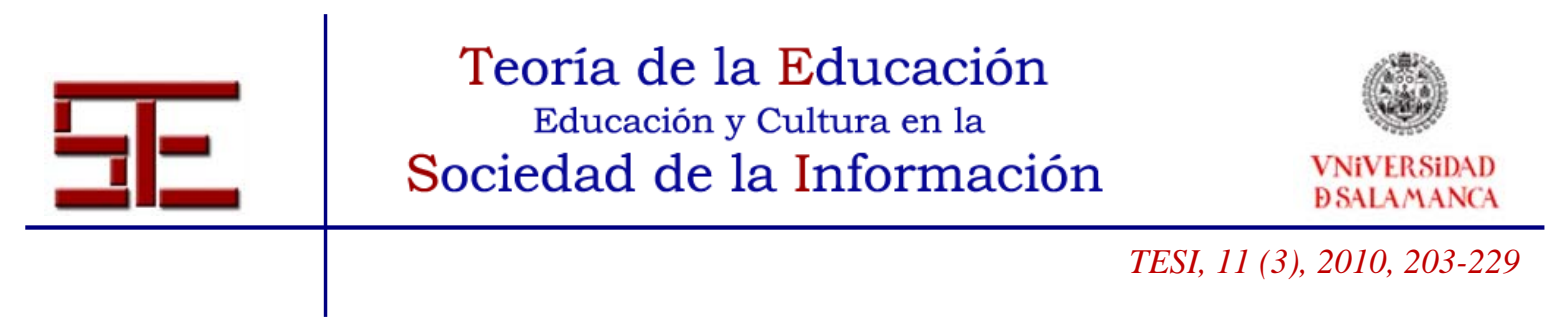

Con frecuencia el enfoque adoptado para la integración de las TIC en el sistema educativo se ha caracterizado por buscar soluciones tecnológicas a problemas pedagógicos. Sin embargo, no está claro que este sea el camino adecuado, si no se introducen otras variables, porque nos enfrentamos a un problema complejo que implica la interacción de múltiples factores y disponemos de pocas reglas que podamos aplicar para todos los casos y contextos. Koehler y Mishra (2008) consideran que una forma fructífera de pensar sobre el complejo problema de enseñar con tecnologías es verlo como un "problema malicioso» (Rittel \& Weber, 1973). Entendiendo por tal, aquel problema cuyas premisas se nos presentan incompletas, llenas de contradicciones y con exigencias cambiantes. Son problemas muy difíciles de resolver e, incluso, de identificar debido a las complejas interdependencias entre un gran número de variables limitadas por el contexto. Si se adopta un proceso lineal de solución se complica su resolución porque la definición del problema, en sí mismo, evoluciona hacia nuevas soluciones. Por lo tanto, es un error enfrentarse a estos problemas de forma convencional. La resolución de uno de sus aspectos puede revelar o crear otro, que incluso sea un problema más complejo. Los problemas «maliciosos» son únicos, novedosos y no tienen una solución «correcta» o «incorrecta», sino simplemente «mejor» o «peor», «suficiente» o «insuficiente». Son los problemas que encontramos en los contextos sociales y, en concreto, en los sistemas educativos. Los diferentes agentes del sistema actúan en este contexto social con diferentes objetivos, expectativas y creencias, lo cual contribuye a aumentar la «maldad» del problema. Los procesos de solución de problemas son cíclicos, no tienen fin y se desarrollan -en el mejor de los casos- en espiral ascendente siendo fuente de nuevos conocimientos. Ahora bien, decir que el proceso de enseñanza-aprendizaje puede ser categorizado como un «problemas malicioso» y complejo, no quiere decir que el problema carezca de estructura porque su complejidad emerge, con frecuencia, de un pequeño grupo de fenómenos tratables y comprensibles que interactúan entre sí.

Una de las principales debilidades de la tecnología educativa es la carencia de una estructura teórica y conceptual para dar forma y guiar la investigación en el ámbito de la enseñanza-aprendizaje con tecnologías. Tras este reconocimiento de la carencia de una orientación teórica para orientar la preparación del profesorado en la integración de las tecnologías, algunos investigadores han iniciado, en los últimos cinco años, esfuerzos de investigación sistemáticos para desarrollar teorías y modelos que consoliden la investigación en el área del pensamiento del profesor sobre la integración de las TIC (Angeli \& Valanides, 2005; Angeli, 2005; Margerum-Lays \& Marx, 2003; Mishra \& Koehler, 2006; Niess, 2005). Estos investigadores defienden la necesidad de desarrollar un nuevo corpus de conocimiento que constituye una extensión del PCK de Shulman (1986, 1987) dentro del dominio de la enseñanza con tecnología. Esta visión extendida del PCK se ofrece como una estructura para revitalizar el estudio del conocimiento del

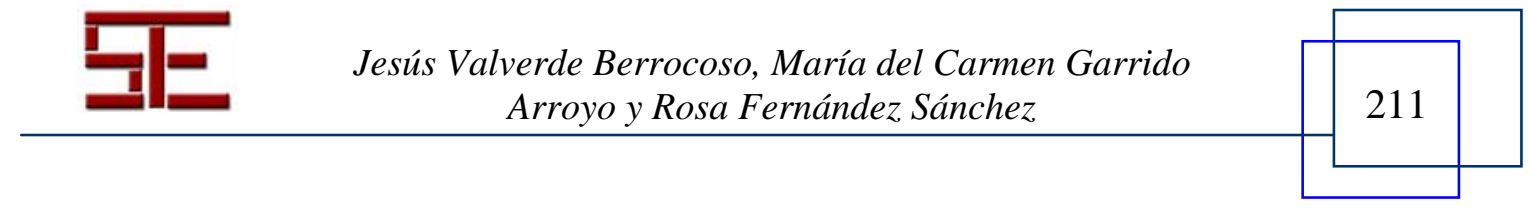




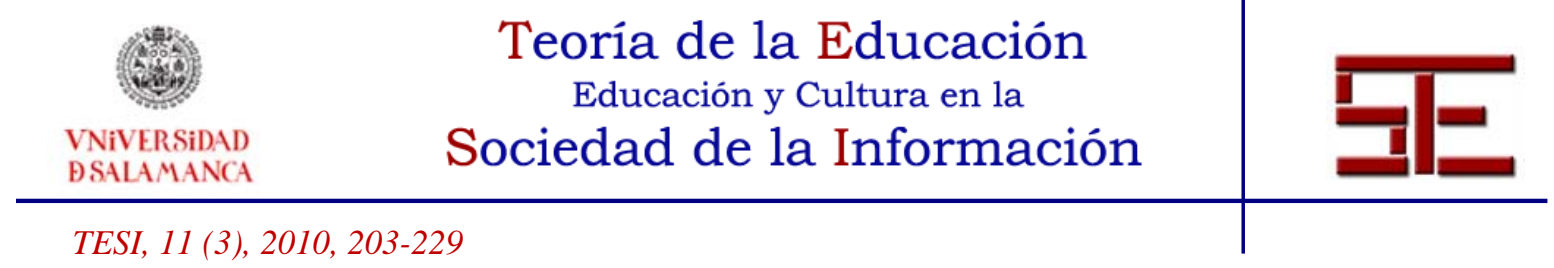

profesor y para recoger y organizar datos sobre el pensamiento del profesor acerca de la integración de la tecnología.

Algunas propuestas, sugeridas en su mayoría por investigadores estadounidenses, de cómo extender el PCK en el ámbito de la enseñanza con tecnología, existen en la literatura bajo diferentes etiquetas. Aquí nos centraremos en el modelo TPCK de Koehler y Mishra (2006, 2008). En cualquier caso, todas las visiones existentes se basan en el principio común: que la efectiva integración de la tecnología presupone una conceptualización que deber ser necesariamente formulada a través de la interacción entre tecnología, contenido curricular y pedagogía.

\section{2.- EL PCK DE SHULMAN}

Para Koehler y Mishra (2006, 2008) en el origen de una buena práctica educativa con TIC existen tres componentes de conocimiento básicos: contenido curricular (CK Content Knowledge), pedagogía (PK - Pedagogical Knowledge) y tecnología (TK Tecnological Knowledge), y las relaciones que se establecen entre ellos. Estas tres bases de conocimiento (CK, PK y TK) forman el núcleo del modelo TPCK. Este enfoque teórico es coherente con otras investigaciones y propuestas teóricas que han intentado ampliar la idea de Shulman sobre PCK (Pedagogical Content Knowledge) al dominio de la tecnología educativa.

El modelo TPCK está construido sobre las aportaciones de Shulman $(1987,1986)$ y, en concreto, basado en su constructo PCK (Pedagogical Content Knowledge) al que se añade el concepto de «Tecnología» $(\mathrm{T})$. Las categorías en las que, según Shulman (1987), se agrupan los conocimientos que un profesor debe dominar son las siguientes: (1) conocimiento del contenido curricular; (2) conocimiento didáctico general, teniendo en cuenta especialmente aquellos principios y estrategias generales de gestión y organización de la clase que trascienden el ámbito de la asignatura; (3) conocimiento del currículo, con un especial dominio de los materiales y los programas que sirven como «herramientas para el oficio del docente»; (4) conocimiento didáctico del contenido, que constituye el conjunto entre materia y pedagogía y manifiesta su forma personal de comprensión profesional; (5) conocimiento de los alumnos y de sus características; (6) conocimiento de los contextos educativos, que abarcan desde el funcionamiento del grupo o de la clase, la gestión y financiación de los distritos escolares, hasta el carácter de las comunidades y culturas, y (7) conocimiento de los objetivos, las finalidades y los valores educativos, así como de sus fundamentos filosóficos e históricos.

Las fuentes principales del conocimiento de los profesores son, para Shulman (1987), la formación académica en la disciplina a enseñar; los materiales y el contexto del proceso

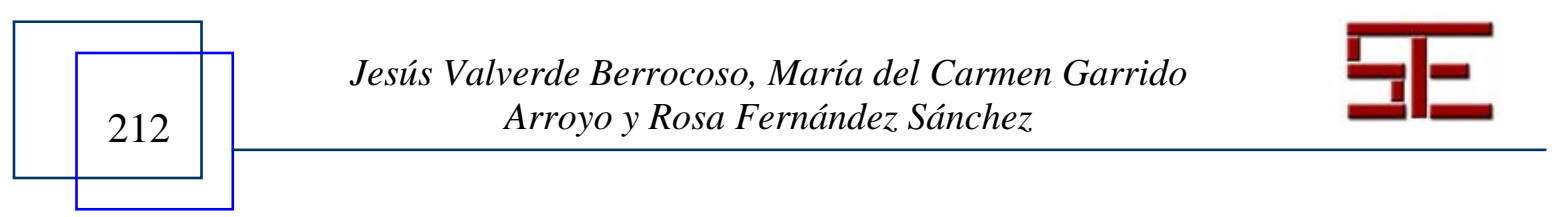




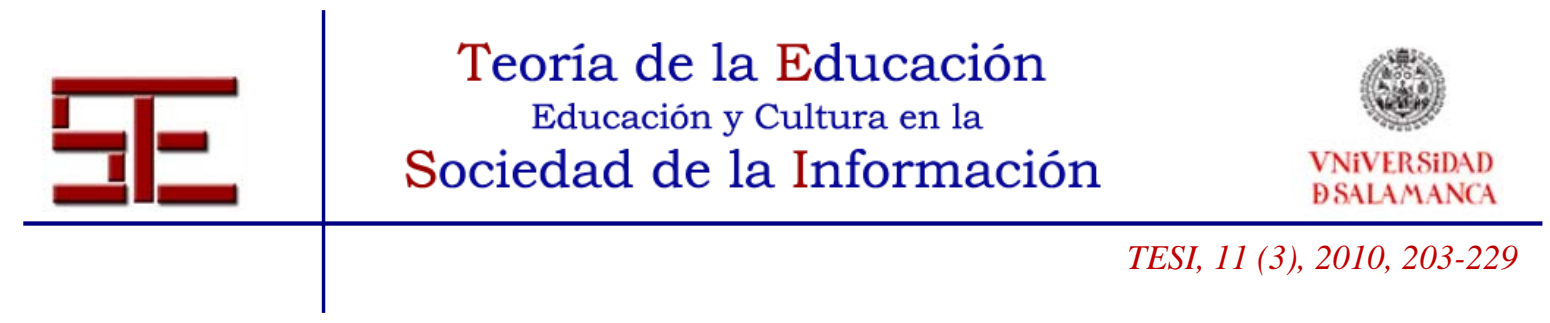

educativo institucionalizado (por ejemplo, los diseños curriculares, los libros de texto, la administración, organización y gestión escolar o la estructura de la profesión docente); la investigación sobre la escolarización, las organizaciones sociales, el aprendizaje humano, la enseñanza y los demás fenómenos socioculturales que influyen en el quehacer de los profesores y, por último, la propia práctica docente.

Para Shulman (2005 19), "enseñar es, en primer lugar, comprender. Le pedimos al maestro que comprenda críticamente un conjunto de ideas que van a enseñarse. Esperamos que entienda lo que enseña y, cuando sea posible, que lo haga de diversas maneras". Pero no basta con la comprensión, el profesor debe orientar su conocimiento hacia la "intersección de la materia y la didáctica" y desarrollar capacidades para "transformar su conocimiento de la materia en formas que sean didácticamente impactantes y aun así adaptables a la variedad que presentan sus alumnos en cuanto a habilidades y bagajes" (Shulman, 2005, 21). Su modelo de acción y razonamiento pedagógico es un ciclo que se compone de actividades de comprensión (inicio y fin del ciclo), transformación, enseñanza, evaluación y reflexión (Ver Fig. 1).

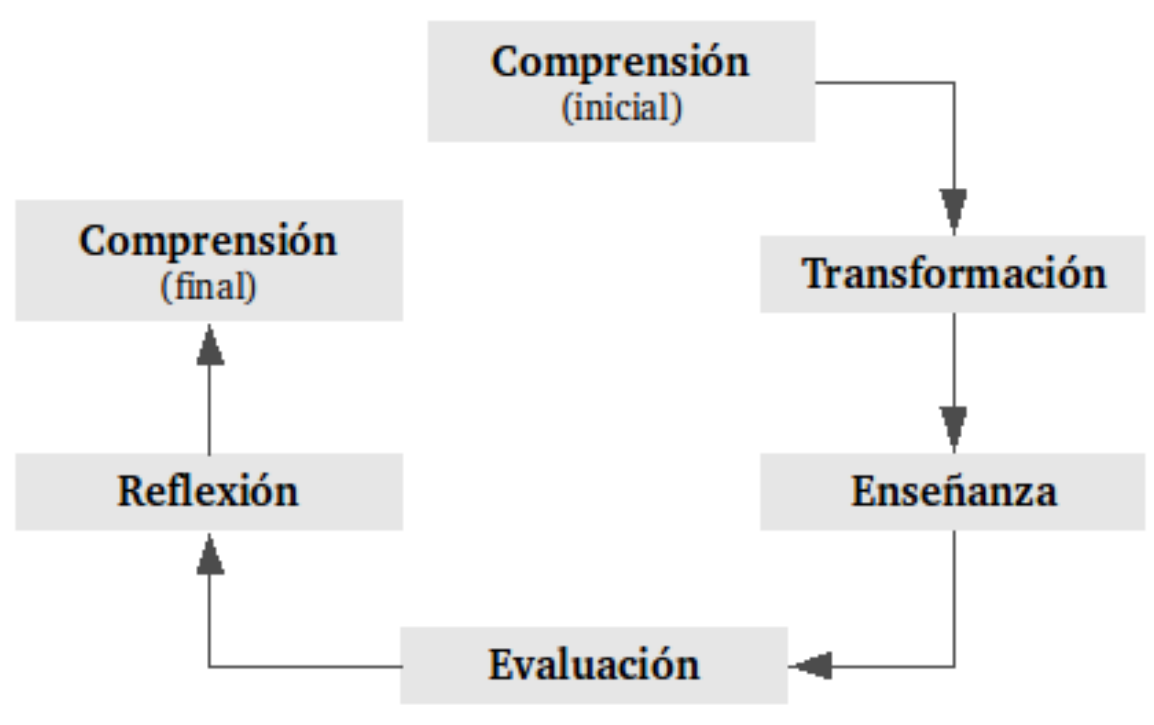

Fig. 1. Ciclo de acción y razonamiento pedagógico de Shulman (1987)

El diseño de la actividad docente exige hacer un recorrido transformador que comienza en la materia tal y como es comprendida por el profesor y acaba en la mente y motivación de los estudiantes. El proceso de transformación conlleva la realización de los siguientes pasos (Shulman, 1987): (1) preparación, que supone la interpretación y

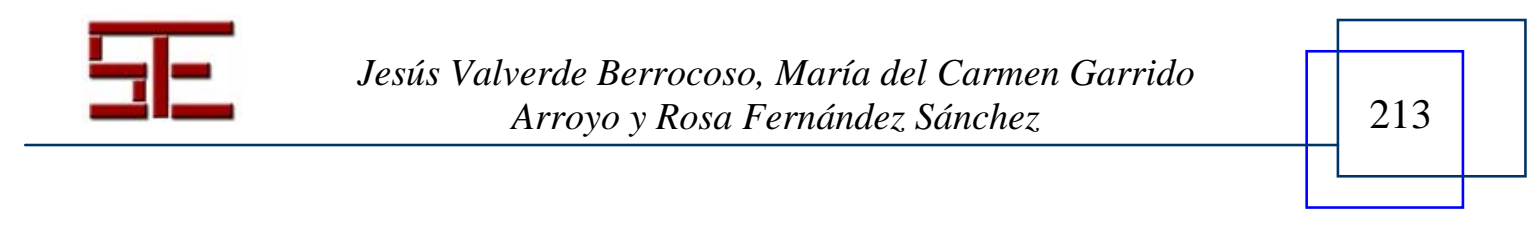




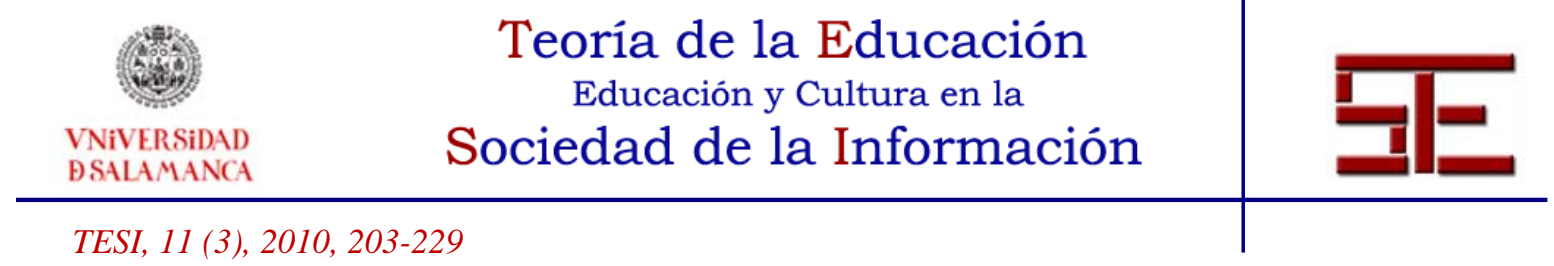

análisis crítico de textos, estructuración y segmentación, creación de un repertorio curricular y clarificación de los objetivos; (2) representación mediante el uso de un repertorio de formas de expresión de conceptos y procedimientos que incluye analogías, metáforas, ejemplos, demostraciones, explicaciones, etcétera; (3) selección, lo cual implica escoger dentro de un repertorio didáctico que incluye métodos de enseñanza, organización y gestión de la actividad docente, y (4) adaptación y ajuste a las características de los alumnos. Esto implica considerar los conceptos, ideas previas, conceptos erróneos y dificultades de aprendizaje, idioma, cultura y motivaciones, clase social, género, edad, capacidad, aptitud, intereses, conceptos de sí mismo y atención. Para Shulman, el razonamiento pedagógico forma parte de la enseñanza en la misma medida que lo hace el acto real de enseñar.

El siguiente momento del ciclo corresponde al acto de impartir enseñanzas. La enseñanza implica, entre otras cosas, la organización y gestión del aula, la presentación del contenido curricular, el establecimiento de interacciones profesor-alumno-medios y la puesta en práctica de metodologías y estrategias didácticas para el aprendizaje. Posteriormente, en la evaluación se verifica la comprensión de los alumnos durante la enseñanza. Se evalúa la comprensión de los estudiantes al finalizar la enseñanza de un contenido y también se evalúa el propio desempeño del profesor y su adaptación a las experiencias del aula. A continuación se produce la reflexión que supone revisar, reconstruir, representar y analizar críticamente el desempeño del profesor y el de la clase, y fundamentar las explicaciones en evidencias. El ciclo concluye con una nueva comprensión de los objetivos, de la materia, de los alumnos, de la enseñanza y del profesor mismo. Se consolidan, de este modo, nuevas maneras de comprender y aprender de la experiencia. Shulman subraya que "la nueva comprensión no se produce automáticamente, ni siquiera después de la evaluación y la reflexión. Para que ella se produzca se necesitan estrategias específicas de documentación, análisis y debate» $(2005,26)$.

Cochran et al. (1993) propusieron una variación a la noción PCK de Shulman basada en una visión constructivista del aprendizaje. Entendían que el concepto «conocimiento» en Shulman era demasiado estático y, por lo tanto, inconsistente con la perspectiva constructivista. Por eso cambiaron PCK por «PCKg» (Pedagogical Content Knowing) para reconocer la naturaleza dinámica del desarrollo del PCK. Y lo definieron como «la integración de la comprensión de un profesor de los cuatro componentes de pedagogía, contenido curricular, características del alumno y contexto de aprendizaje» (p. 266) y enfatizaron la naturaleza interaccional del PCK, como resultado del desarrollo concurrente de estos cuatro componentes. Las flechas de la figura representan el desarrollo del PCKg como resultado de nuevas experiencias y actividades de aprendizaje.

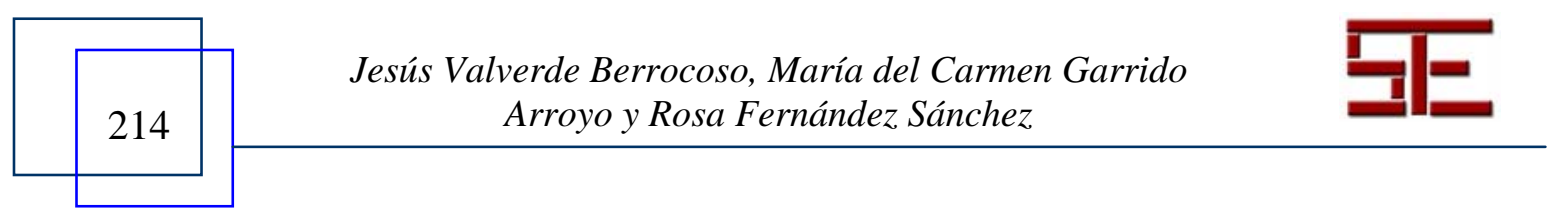



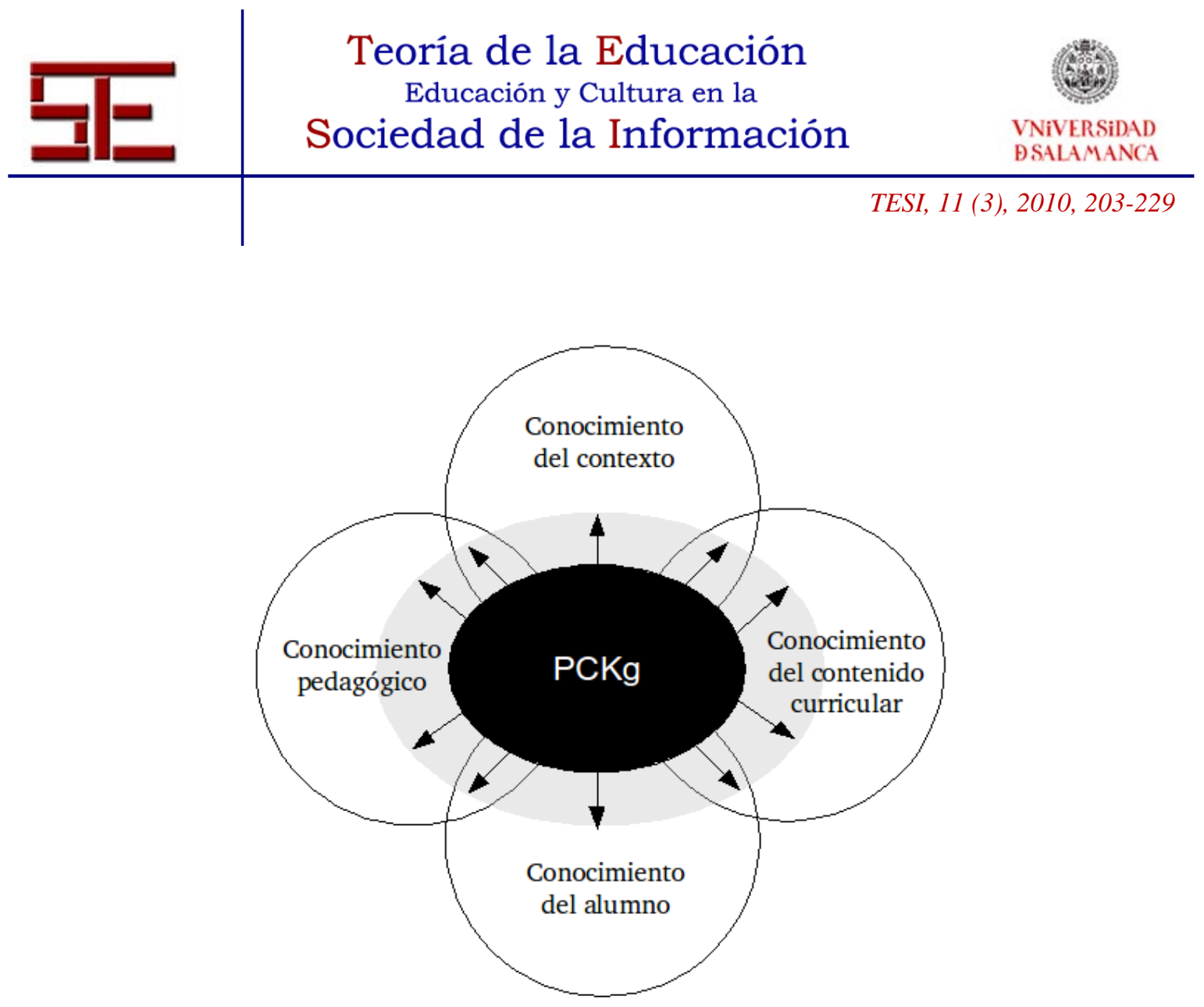

Fig. 2. El modelo PCKg adoptado por Cohran et al. (1993)

En general, no existe una conceptualización universalmente aceptada del PCK. Las principales diferencias entre los expertos tienen que ver con los elementos incorporados al PCK y las denominaciones específicas con las que describen a esos elementos. Sin embargo, todos los investigadores incluyen en el constructo el PCK tanto el conocimiento del profesor sobre las representaciones de su asignatura como su conocimiento de las concepciones del alumno y las dificultades relacionadas con el contenido de la materia. Existe también acuerdo en que el PCK tiene que ver específicamente con la enseñanza de un contenido particular y diferencia entre conocimiento general de carácter pedagógico, el conocimiento de los objetivos educativos y las características del alumno. Por último, hay acuerdo en que el PCK es un conocimiento profundamente radicado en la práctica de aula y que se desarrolla, gradualmente, con nuevas experiencias de enseñanza. Esto implica que los profesores noveles tiene unos niveles incompletos y superficiales del PCK y que su conocimiento crece continuamente con nuevas experiencias relacionadas con la enseñanza y el aprendizaje (Angeli \& Valanides, 2009).

Shulman (1986) no incluyó explícitamente en su modelo la tecnología y sus relaciones con el contenido, la pedagogía y los alumnos y, por consiguiente, en su forma original

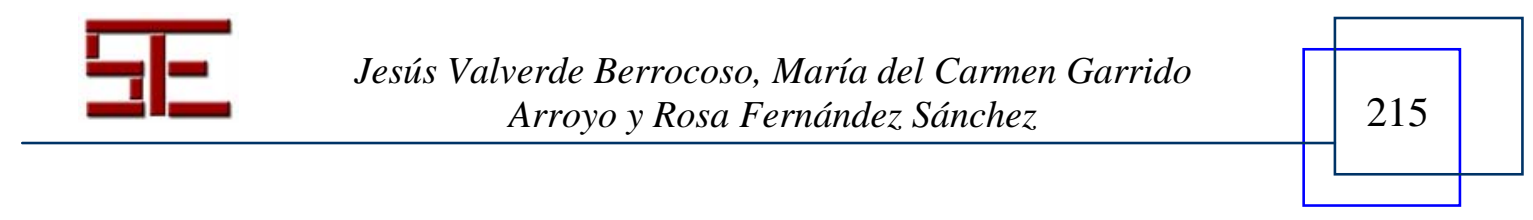




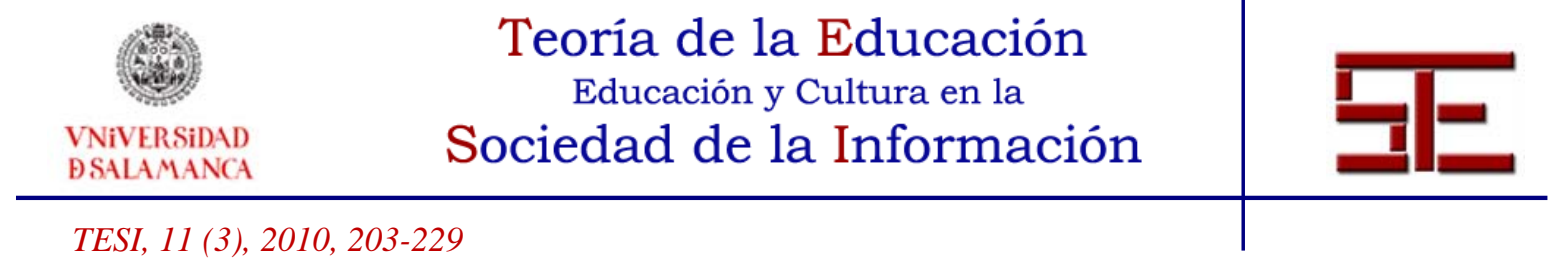

no explicó cómo los profesores usan las posibilidades de la tecnología para transformar el contenido y la pedagogía para sus alumnos. En la actualidad, con el continuo incremento de ordenadores en las aulas y las numerosas aplicaciones de las TIC, el constructo PCK necesita ser ampliado para tomar en consideración las necesidades para estudiar y comprender el pensamiento del profesor sobre el uso educativo de los ordenadores.

\section{3.- EL MODELO TPCK DE KOEHLER Y MISHRA}

La base de este modelo teórico es la comprensión de que la enseñanza es una actividad altamente compleja que precisa de muchos tipos de conocimiento. Es una habilidad cognitiva compleja que tiene lugar en un contexto poco estructurado y dinámico. La idea del TPCK (no así el término) ha tenido diferentes precursores. Pierson (2001) adelantó, de modo casi exacto, la actual conceptualización diagramática del TPCK. Zhao (2003) también describe los componentes del TPCK, mientras que otros autores han utilizado ideas similares, aunque con diferentes enfoques y terminología. Entre éstas se encuentran el ICT-PCK (Angeli \& Valanides, 2009) que comentaremos más adelante. Mishra y Koehler lo formularon en 2006 y han continuado en los años siguientes investigando y publicando sobre este modelo teórico de integración de las TIC para la buena práctica educativa.

Lo que ofrece como novedad este enfoque es la articulación de las relaciones entre Contenido Curricular, Tecnología y Pedagogía. En términos prácticos esto significa que, además de considerar cada uno de estos componentes de manera aislada, necesitamos considerarlos por pares y los tres en conjunto. Es similar al planteamiento realizado por Shulman, en el que consideró las relaciones entre C y $\mathrm{P}$ y lo denominó PCK. En este caso, una consideración similar conduce a tres intersecciones con pares de conocimientos y a una tríada. Uno de los pares, PCK, fue introducido y articulado por Shulman, y Koehler y Mishra (2006) introducen dos nuevos pares y una tríada. Así pues, en el modelo TPCK hay tres componentes principales de conocimiento: Contenido Curricular, Pedagogía y Tecnología. Con igual importancia en el modelo están las interacciones entre estos corpus de conocimiento, representados como Conocimiento de la Pedagogía y el Contenido Curricular (PCK), Conocimiento de la Tecnología y el Contenido Curricular (TCK), Conocimiento de la Pedagogía y la Tecnología (TPK) y Conocimiento de la Pedagogía, el Contenido Curricular y la Tecnología (TPCK) (ver Fig. 3).

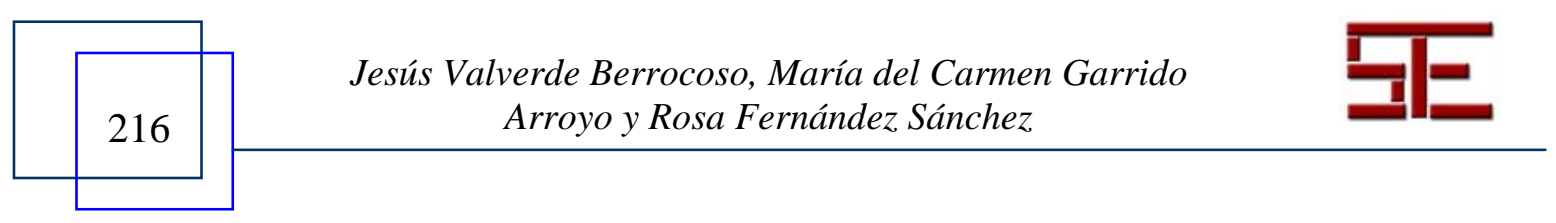



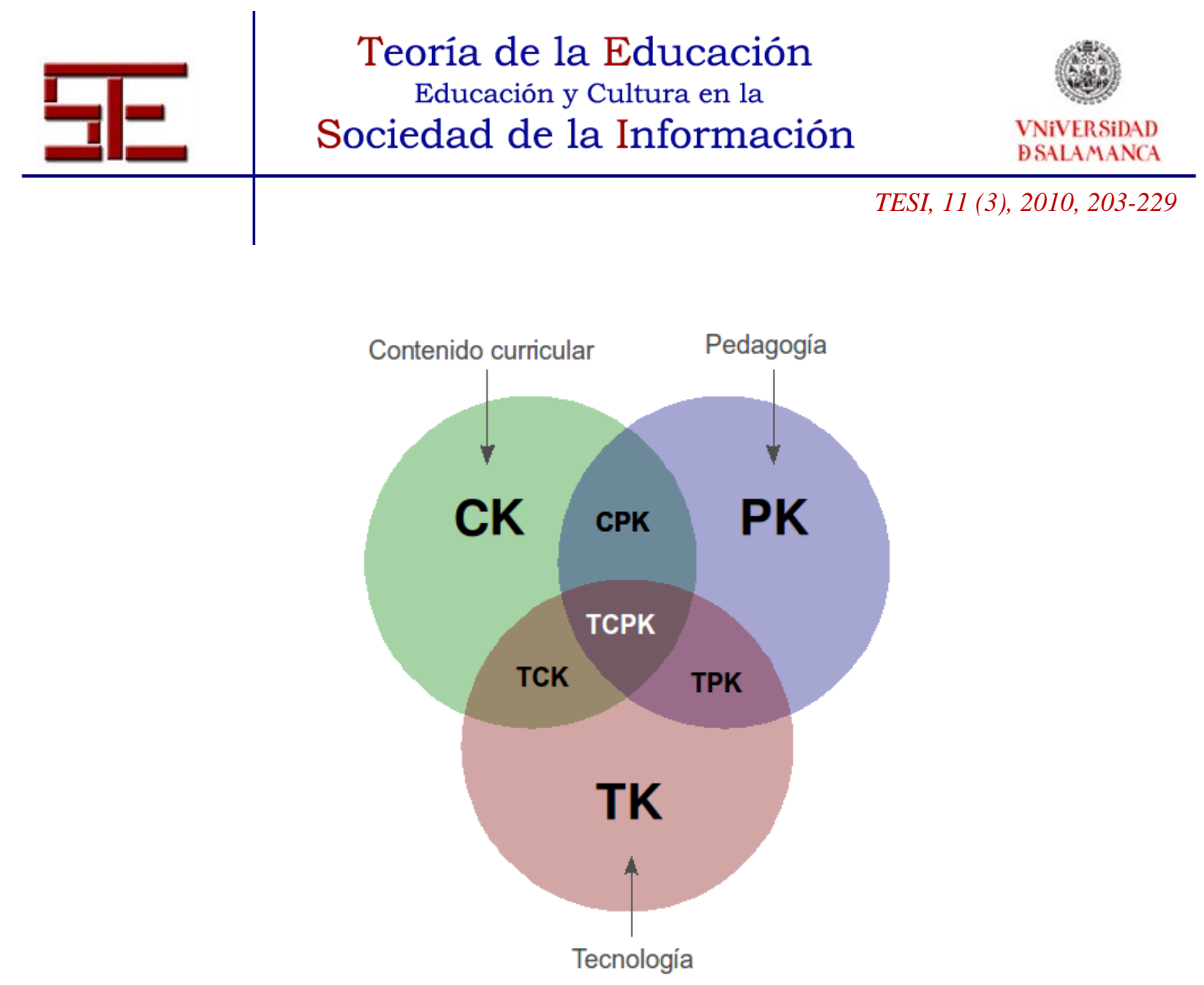

Fig. 3. Estructura TPCK y sus componentes de conocimiento (Koehler \& Mishra, 2008, 12)

En este modelo teórico, el concepto «conocimiento» adopta un enfoque pragmático bajo las premisas de autores como Dewey, Schön y Perkins (Dewey, 2002; Perkins, 1986; Schön, 1998, 2008). Para este último, el conocimiento puede ser considerado como una herramienta que está diseñada y adaptada a un propósito. «Pensar el conocimiento como diseño es concebirlo como un utensilio que uno construye y empuña más que como un objeto dado para observarlo y descubrirlo» (Perkins, 1986, 132). Lo importante es la utilidad del conocimiento (knowledge usable) (Kelly, 2003).

A continuación se describen cada uno de los conocimientos que constituyen el modelo teórico TPCK de Koehler y Mishra (2006, 2008).

\section{1.- Los componentes del modelo TPCK}

El conocimiento del Contenido Curricular (CK) es el conocimiento sobre el área de conocimiento, asignatura o disciplina que se enseña y se aprende. Este conocimiento es fundamental para un profesor. Según Shulman (1986) incluye los conocimientos de conceptos, teorías, ideas, estructuras organizativas, evidencias y pruebas, así como prácticas establecidas y enfoques sobre el desarrollo de dicho conocimiento. El

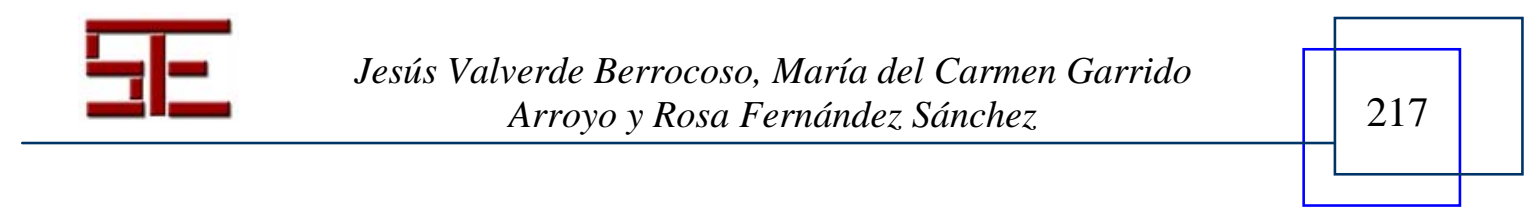




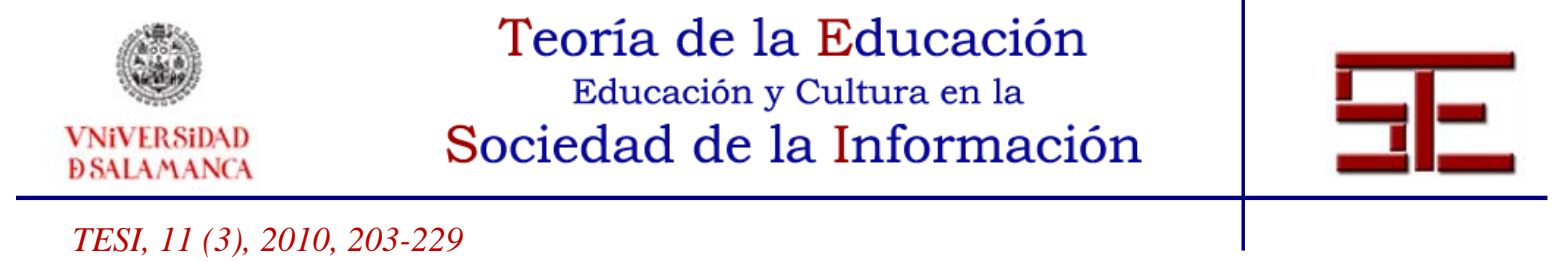

conocimiento y la naturaleza de la investigación difieren enormemente entre campos y es importante para los profesores comprender los fundamentos profundos del conocimiento de la disciplina que enseñan. El coste de no poseer una base comprehensiva del conocimiento del contenido curricular puede ser bastante alto; los alumnos pueden recibir una información incorrecta y desarrollar errores conceptuales sobre el contenido de aprendizaje. Las diferencias entre diversos grados de competencia sobre el conocimiento del contenido curricular reflejan diferentes estrategias para integrar las TIC en la actividad docente y definen el grado de una buena práctica educativa con tecnologías.

El conocimiento de la Pedagogía (PK) es un conocimento profundo sobre los procesos y prácticas o métodos de enseñanza y aprendizaje e incluye (entre otras cosas) a los objetivos generales, valores y metas de la educación. Es una forma genérica de conocimiento que se aplica al aprendizaje del alumno, la gestión u organización escolar, la planificación docente y el desarrollo curricular o la evaluación de los aprendizajes. Incluye conocimientos sobre estrategias didácticas o métodos de enseñanza aplicados en el aula, la naturaleza de los destinatarios de la acción formativa o las técnicas para evaluar la comprensión del alumno. Un profesor con un profundo conocimiento pedagógico comprende cómo el estudiante construye el conocimiento y adquiere competencias o cómo desarrolla estrategias cognitivas y una adecuada motivación hacia el aprendizaje. El conocimiento de la Pedagogía requiere una comprensión de teorías sobre el desarrollo humano, teorías cognitivas y sociales y cómo aplicarlas a los alumnos en el aula.

El conocimento de la Tecnología (TK) es un tipo de conocimiento que está constantemente en un estado de cambio continuo, más que los otros dos conocimentos centrales de la estructura TPCK (Pedagogía y Contenido Curricular). En cualquier caso, Koehler y Mishra $(2006,2008)$ creen que existen ciertas formas de «pensamiento sobre» y de «trabajo con» la Tecnología que puede aplicarse a todas las herramientas tecnológicas. En este sentido, su definición de TK se aproxima al concepto de «fluidez de la Tecnología de la Información» (FITness) propuesta por el Comité para la Alfabetización en Tecnologías de la Información del National Research Council (NRC, 1999). El FITness va más allá de la tradicional noción de alfabetización computacional porque requiere que las personas comprendan las tecnologías de la información de forma suficientemente amplia y global, como para ser capaces de aplicarlas, de modo productivo, al trabajo y a su vida cotidiana; saber reconocer cuándo las tecnologías de la información pueden ayudar o impedir el logro de una meta y saber adaptarse continuamente a los cambios en estas tecnologías. FITness requiere, por consiguiente, una comprensión más profunda y un mayor dominio de las tecnologías para procesar la información, la comunicación y la solución de problemas que las que se derivan de la definición tradicional de alfabetización informacional. La adquisición de TK permite a

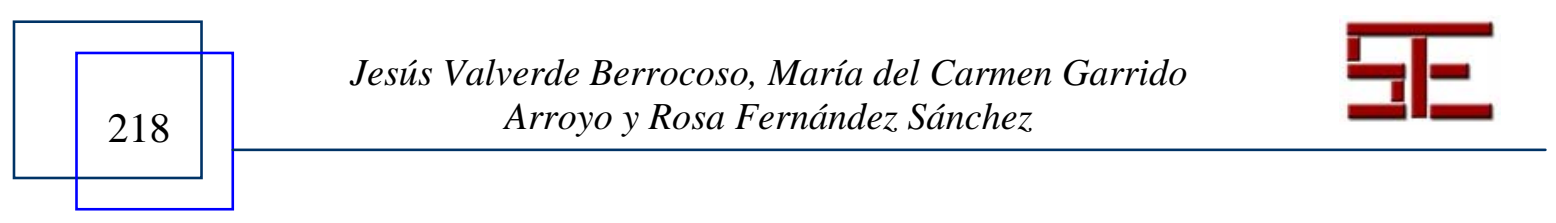




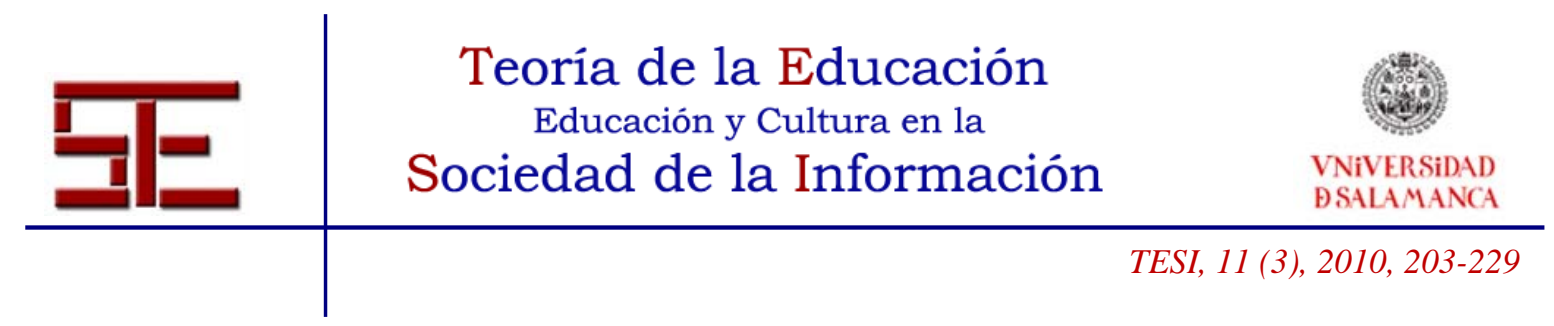

las personas llevar a cabo una variedad de tareas diversas usando las TIC y desarrollando diferentes formas de llevar a cabo una tarea dada. Esta conceptualización de TK no establece un estado «final», sino más bien un estado «en desarrollo» continuo.

El conocimiento de la Pedagogía y del Contenido Curricular (PCK) es coherente con la idea de Shulman y aplicable a la enseñanza de un contenido específico. PCK se ocupa de aspectos que son claves para una buena práctica educativa: conciencia de los errores conceptuales más comunes y formas de descubrirlos; la importancia de avanzar enlaces y conexiones entre diferentes ideas del contenido curricular; el conocimiento previo del alumno; estrategias alternativas de enseñanza o la flexibilidad para explorar formas alternativas de comprender una misma idea o problema. Para Shulman, la noción central de este conocimiento es la transformación de la materia para la enseñanza. Esta transformación tiene lugar cuando el profesor interpreta la disciplina, encuentra múltiples formas para representarla y adapta los materiales didácticos a concepciones alternativas y al conocimiento previo del alumno.

El conocimiento de la Tecnología y el Contenido Curricular (TCK) es una comprensión de la forma en la que la tecnología y el contenido curricular se influyen y limitan el uno al otro. Comprender el impacto de la tecnología sobre las prácticas y el conocimiento de una determinada disciplina es básico si queremos desarrollar apropiadas herramientas tecnológicas con fines educativos. La elección de las tecnologías posibilita y limita los tipos de conceptos que pueden ser enseñados. De la misma forma, determinadas decisiones sobre el contenido pueden limitar los tipos de tecnologías que pueden ser utilizado. La tecnología limita los tipos de posibles representaciones pero, también, posibilita la construcción de nuevas y variadas representaciones. Además, las herramientas tecnológicas pueden proporcionar un mayor grado de flexibilidad al moverse a través de estas representaciones. Los profesores necesitan dominar algo más que la materia que ellos enseñan, deben poseer también una profunda comprensión de la forma en que su disciplina (o las clases de representación que pueden ser construidas) puede ser cambiada al aplicar las tecnologías. El profesor necesita comprender qué tecnologías específicas son las mejor situadas para ser utilizadas en su contenido de aprendizaje y cómo el contenido curricular dicta o, quizá, cambia la tecnología, y viceversa. Para Koehler y Mishra (2006, 2008) el TCK es el conocimiento más desatendido entre las diferentes intersecciones del modelo TPCK. La estructura del TCPK sugiere que las experiencias de los profesores con las tecnologías necesita ser específica para diferentes áreas de contenidos curriculares.

El conocimiento de la Tecnología y la Pedagogía (TPK) es una comprensión acerca de cómo cambian la enseñanza y el aprendizaje cuando se utilizan determinadas tecnologías. Incluye el conocimiento de las posibilidades y limitaciones pedagógicas del conjunto de herramientas tecnológicas a utilizar en los contextos de aprendizaje de

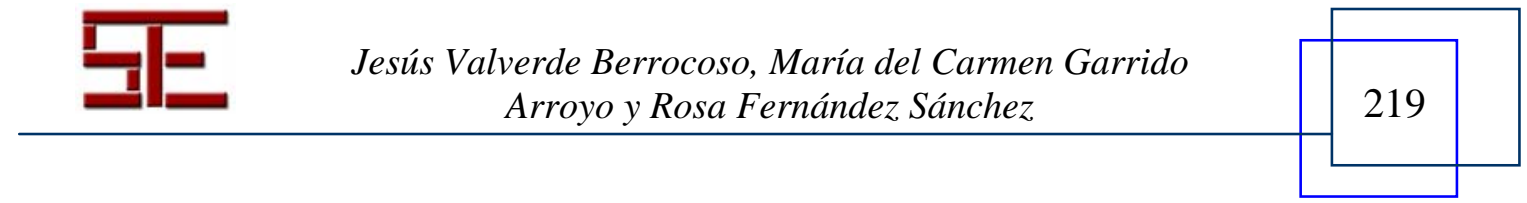




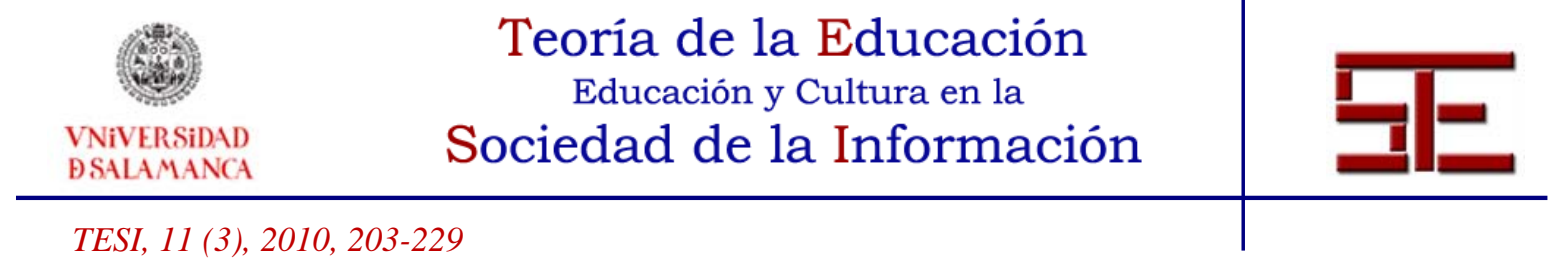

disciplinas específicas. Una misión importante del TPK es el desarrollo de la flexibilidad creativa con las herramientas disponibles para reformular sus propósitos técnicos a finalidades educativas específicas. En el terreno del software la mayoría de los programas son diseñados para contextos no educativos. Los profesores necesitan desarrollar habilidades que superen la propia tecnología y la «reconfiguren» de manera creativa para sus propósitos pedagógicos.

Por último, la integración de todos los conocimientos da lugar al TPCK, que Koehler y Mishra (2006, 2008) consideran como una forma emergente de conocimiento que va más allá de los tres conocimientos básicos (Contenido Curricular, Pedagogía y Tecnología). Es una comprensión que emerge desde la interacción de los tres componentes esenciales del modelo. Es la base de una buena práctica educativa con TIC y exige una comprensión de la representación de conceptos cuando usamos determinadas tecnologías; de las técnicas pedagógicas que usan las tecnologías de forma constructiva para enseñar un contenido curricular; del conocimiento sobre lo que hace un concepto fácil o difícil de aprender y cómo las tecnologías pueden ayudar a compensar (o reparar) algunas de las dificultades de aprendizaje a los que los estudiantes se enfrentan; el conocimiento previo del alumno y las teorías epistemológicas del profesor, así como los conocimientos sobre cómo las tecnologías pueden ser utilizadas para construir nuevos conocimientos a partir del conocimiento preexistente y desarrollar nuevas epistemologías o mejorar las antiguas.

El TPCK es una forma de conocimiento que los profesores expertos ponen en juego en cualquier momento de su actividad docente. Cada «problema malicioso» que genera la práctica de aula se presenta a los profesores como una combinación única de estos tres factores $\mathrm{y}$, en consecuencia, no existe una solución tecnológica simple para cada situación educativa. Más bien, las soluciones se hallan en la habilidad del profesor para navegar con flexibilidad en el espacio definido por los tres elementos, y sus complejas interacciones, en unos contextos específicos. Ignorar la complejidad inherente a cada componente del conocimiento, o la complejidad de las relaciones entre estos componentes, puede conducir a una simplificación de las soluciones o de los errores. Por eso, los profesores no sólo necesitan desarrollar una flexibilidad cognitiva para cada uno de estos conocimientos básicos ( $\mathrm{T}, \mathrm{P}$ y $\mathrm{C}$ ), sino también sobre la manera en la que estos dominios interactúan, de modo que puedan encontrar soluciones que sean sensibles a los contextos específicos.

Separar los tres componentes de conocimiento es un proceso analítico que no funciona para alcanzar la comprensión de la práctica. Ver cualquiera de estos componentes de manera aislada de los otros representa un perjuicio real para la buena práctica educativa con TIC. La enseñanza y el aprendizaje con tecnologías existe en una transacción dinámica de relaciones entre los tres componentes de la estructura. Un cambio en

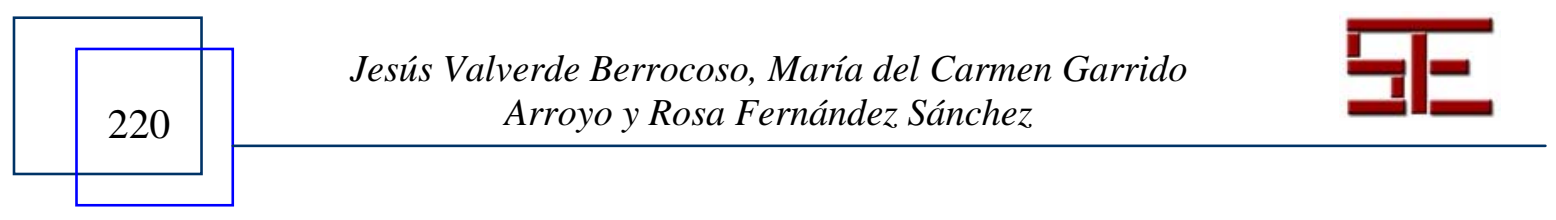




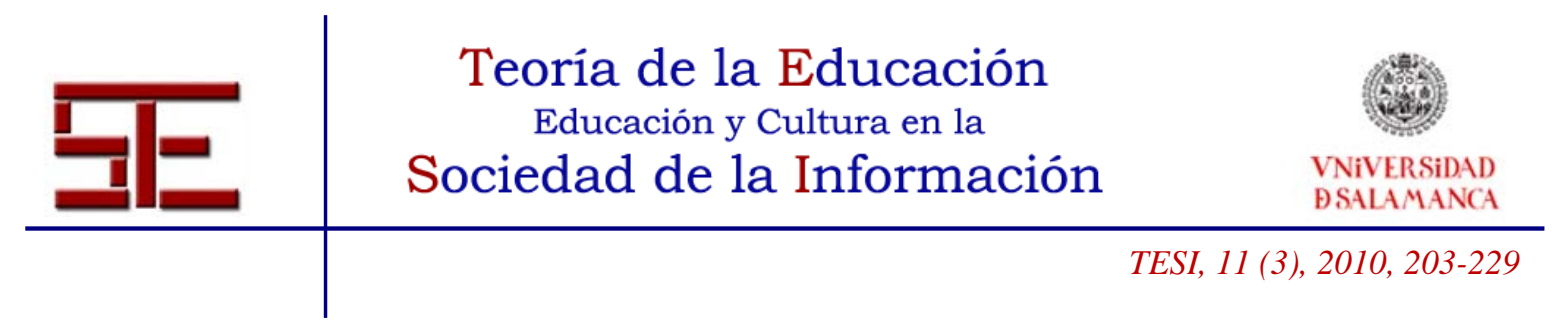

cualquiera de estos factores tiene que ser compensado por cambios en los otros dos (Mishra \& Koeler, 2006).

Esta compensación se hace más evidente cuando una nueva tecnología educativa obliga a los profesores a reformular sus presupuestos pedagógicos y deben reconstruir el equilibrio dinámico entre los tres elementos. De ese modo se invierte la perspectiva convencional de que el contenido curricular simplemente necesita ser convertido para «encajar» en la nueva tecnología. La introducción de Internet, particularmente la aparición del e-learning en la educación secundaria y universitaria, es un ejemplo de la llegada de una tecnología que fuerza al profesorado a reflexionar sobre asuntos básicos de la educación, tanto en el ámbito disciplinar como en el exclusivamente pedagógico. Por otra parte, la llegada de hipertextos desarrollados por comunidades de usuarios en Internet y de enciclopedias on-line, el uso del bookmarking social y su utilización en sitios webs 2.0 tan populares como Wikipedia, Delicious, YouTube o Flickr, ha abierto los ojos a muchos profesores que ahora se dan cuenta del poder constructivista de las folksonomías y otros esquemas de categorización, para reconfigurar cómo comprendemos textos y las relaciones entre ellos. Para Koehler y Mishra (2008) enseñar con tecnologías es una actividad difícil de llevar a cabo. El modelo TCPK sugiere que $\mathrm{C}$, P y T tienen papeles que jugar de modo individual y colectivo. Enseñar eficazmente con tecnología requiere continuamente crear, mantener y restablecer un equilibrio dinámico entre cada componente.

El TPCK define el corpus de conocimiento que los profesores necesitan para enseñar «con» y «sobre» tecnología en sus diferentes asignaturas, niveles y cursos. Según Niess (2008), el TPCK es algo más que un conjunto de múltiples dominios de conocimiento y competencias que los profesores necesitan para enseñar a sus alumnos determinadas asignaturas en un curso específico. TPCK es una forma de reflexión dentro de estos múltiples dominios de conocimiento. Los tipos de conocimiento que están implicados en TPCK son los siguientes (Shavelson et al., 2003): declarativo (saber qué, lo cual incluye definiciones, términos, hechos y descripciones), procedimental (saber cómo, es decir, secuencias de pasos para completar una tarea o subtarea), esquemático (saber por qué; se extrae de los dos anteriores conocimientos y crea principios y modelos mentales) y estratégico (saber cuándo y dónde usar un determinado conocimiento y estrategias como planificación y solución de problemas junto con el control del progreso hacia una meta).

Así pues, ¿qué es lo que necesita saber un futuro maestro dentro del modelo TPCK en un aula tecnológica? En primer lugar, comprender la diversidad de alumnos y sus necesidades de aprendizaje. También planificar y diseñar entornos de aprendizaje y experiencias que satisfagan la diversidad de necesidades de aprendizaje de los alumnos. En tercer lugar, desarrollar estrategias didácticas eficaces para atender adecuadamente a

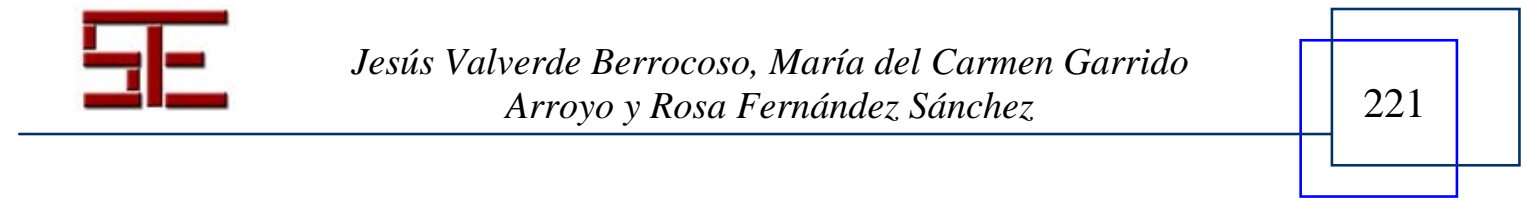




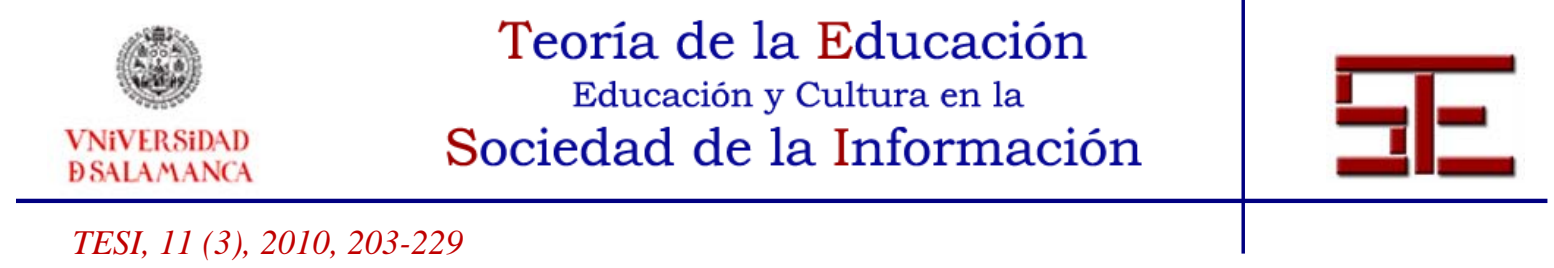

la diversidad de necesidades del aprendizaje de los alumnos. Además, identificar estrategias de gestión de aula para apoyar la diversidad de alumnos en un aula tecnológica y, por último, evaluar a la diversidad de estudiantes en una aula mediada por tecnologías (Niess, 2008). La complejidad en la toma de decisiones que implica la adopción del modelo TPCK puede observarse en la Fig. 3, que recoge la representación de un proceso de toma de decisiones para una actividad de aula con TIC (Schmidt \& Gurbo, 2008, 73).

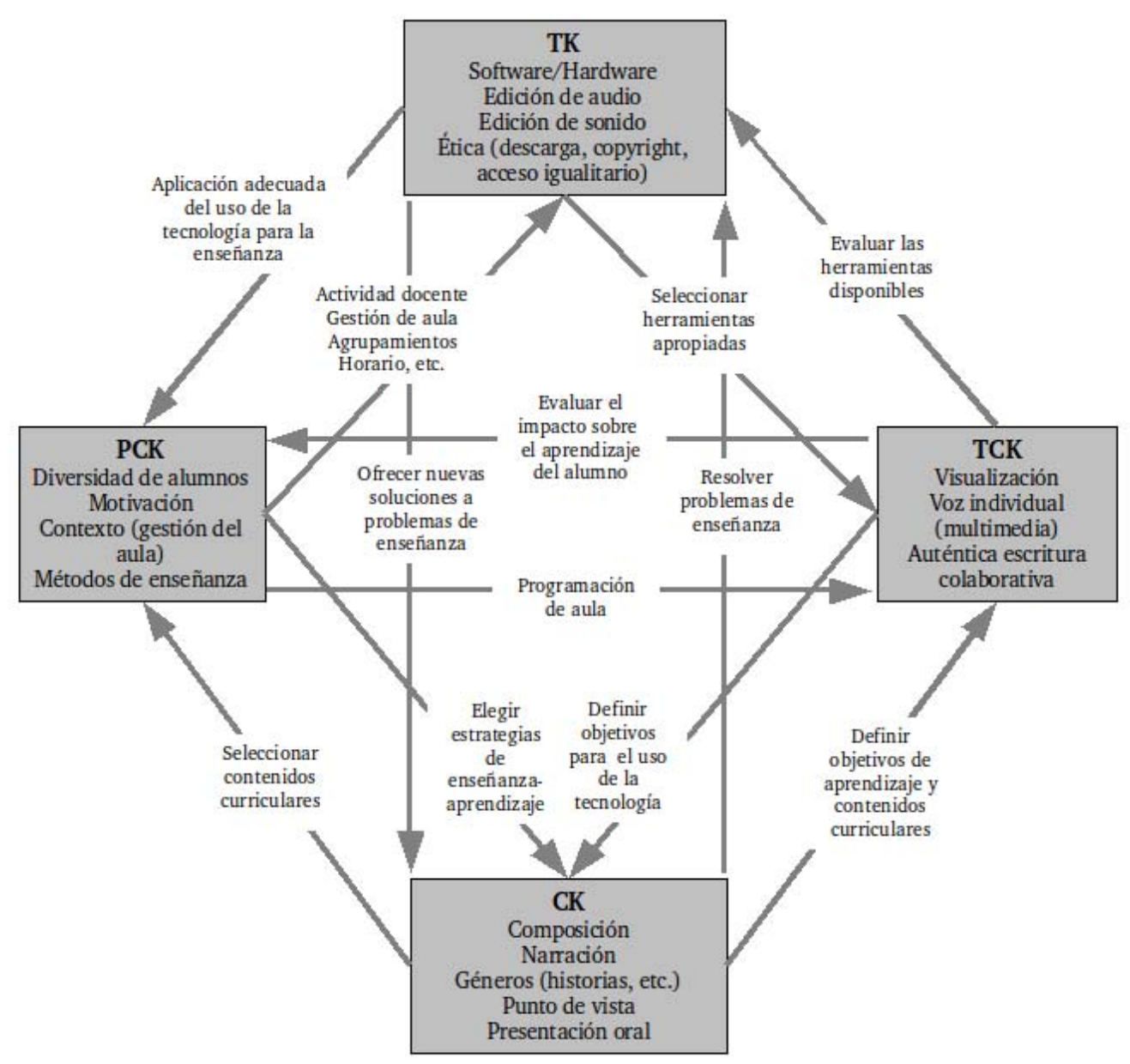

Fig. 4. Proceso de toma de decisiones relacionado con TPCK usado para crear historias narrativas en formato digital (Schmidt \& Gurbo, 2008, 73)

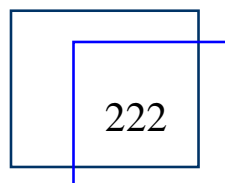

Jesús Valverde Berrocoso, María del Carmen Garrido Arroyo y Rosa Fernández Sánchez 


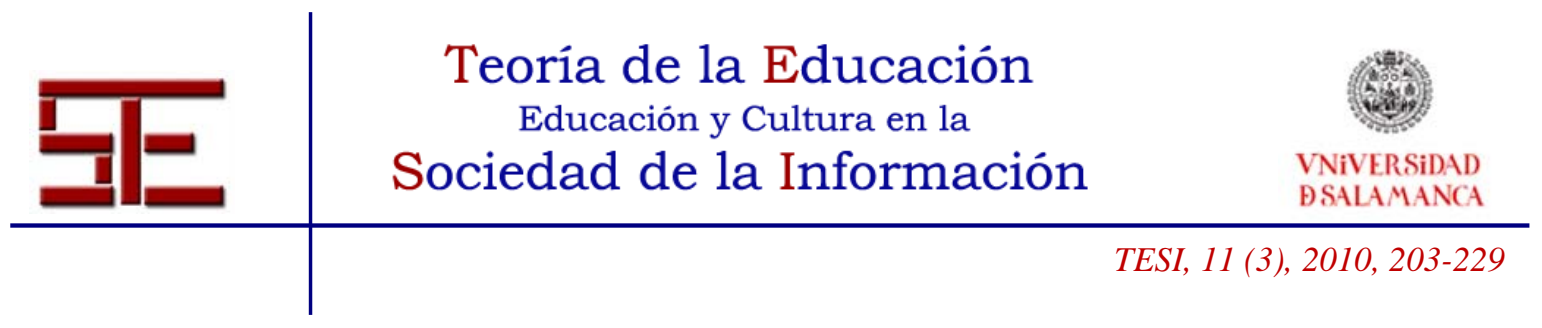

\section{2.- El modelo ICT-TPCK de Angeli y Valadines}

Para Angeli \& Valanides (2009) un tema que los investigadores no han desarrollado correctamente en el modelo TPCK es si el constructo es un corpus de conocimiento diferenciado o único que está construido desde otras formas de conocimiento del profesor (lo que ellos denominan el punto de vista transformador), o si el TPCK no es una forma de conocimiento diferenciado, sino que está integrado desde otras formas de conocimiento del profesor «en el acto docente», durante su actividad educativa (denominado, el punto de vista integrador).

Aunque la literatura actual sobre el TPCK muestra cómo la mayoría de los investigadores consideran que el crecimiento de cualquiera de los constructos relacionados (CK, PK y TK) automáticamente contribuye al desarrollo en el TPCK (Koehler et al., 2007), otros como Angeli \& Valadines (2009) lo ponen en duda a la luz de sus propias investigaciones. Durante los últimos cinco años, han llevado a cabo un número de investigaciones empíricas considerando los usos educativos de los ordenadores y basándose en estos descubrimientos concluyen que el crecimiento en los constructos relacionados no significa automáticamente un crecimiento en el TPCK.

Estos hallazgos sugieren que el TPCK en sí mismo es un cuerpo de conocimiento diferente de sus componentes constitutivos (Angeli \& Valanides, 2005; Angeli, 2005; Valanides \& Angeli, 2006, 2008a, 2008b). En particular, los profesores en activo, que tienen una amplia experiencia docente y conocimientos de diversos programas informáticos, pero que no fueron formados específicamente sobre cómo enseñar con ordenadores, no llevan a cabo -de modo significativo- una mejor práctica en lecciones mediadas por ordenador para sus alumnos que otros profesores, que tenían menos experiencia docente, buenas competencias informáticas, pero tampoco habían recibido formación específica en los usos educativos de los ordenadores (Valanides \& Angeli, 2008b). Sin embargo, después de una formación específica sobre cómo enseñar con ordenadores, los profesores con competencias pedagógicas más desarrolladas y mejor conocimiento sobre el contenido y los alumnos sobresalieron por encima de otros profesores con menos conocimiento en esas áreas (Valanides \& Angeli, 2008c). Resultados similares se encontraron con estudiantes de Magisterio. Estudios con estudiantes universitarios mostraron que sin una formación específica en el uso educativo de ordenadores, alumnos de $2^{\circ}$ y $3^{\circ}$ que tenían buenas competencias informáticas no diseñaron mejor lecciones mediadas por ordenador que alumnos de $1^{\circ}$ con buenas competencias informáticas pero sin formación específica en el uso educativo de los ordenadores. Sin embargo, después de esta formación, alumnos de $2^{\circ}$ y $3^{\circ}$ sobresalieron por encima de los alumnos de $1^{\circ}$ en el diseño de actividades de

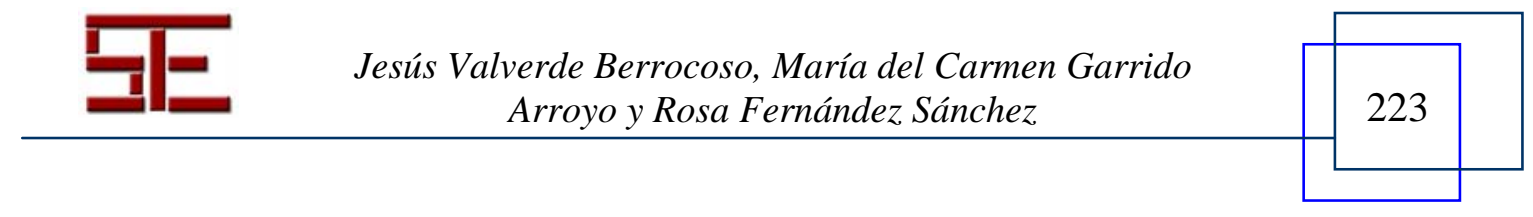




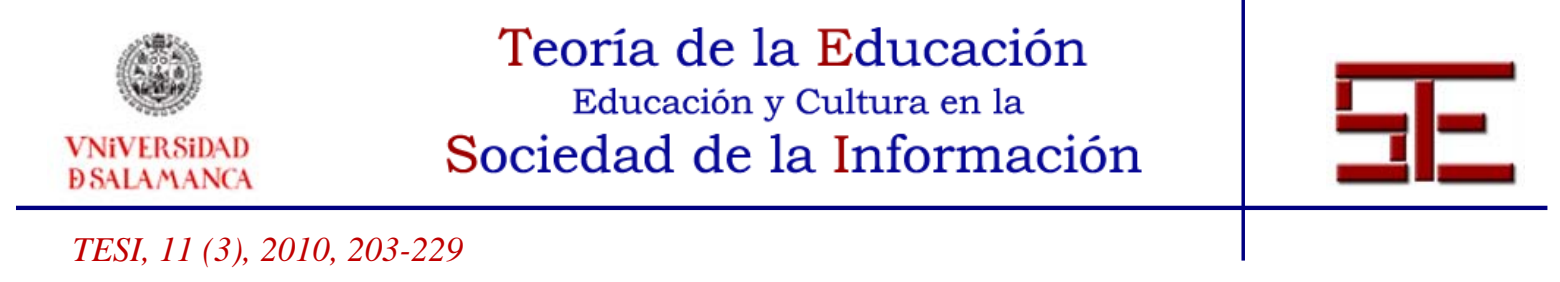

aprendizaje con ordenadores (Angeli \& Valanides, 2005; Angeli, 2005; Valanides \& Angeli, 2006, 2008a).

A partir de los resultados de sus investigaciones empíricas, los autores sugieren que el TPCK es un corpus de conocimiento diferenciado que puede ser desarrollado y evaluado. Este corpus va más allá de la mera integración o acumulación de los conocimientos base que lo constituyen, y se orienta la transformación de estos conocimientos en algo nuevo. Así, los autores no apoyan la visión integradora, ya que el crecimiento del conocimiento base a partir de la contribución individual, sin formación específica dirigida exclusivamente al desarrollo del TPCK, no conlleva ningún crecimiento en el TPCK (Angeli \& Valanides, 2005; Angeli, 2005; Valanides \& Angeli, 2006, 2008a, 2008b), indicando claramente que el TPCK es un único corpus de conocimiento.

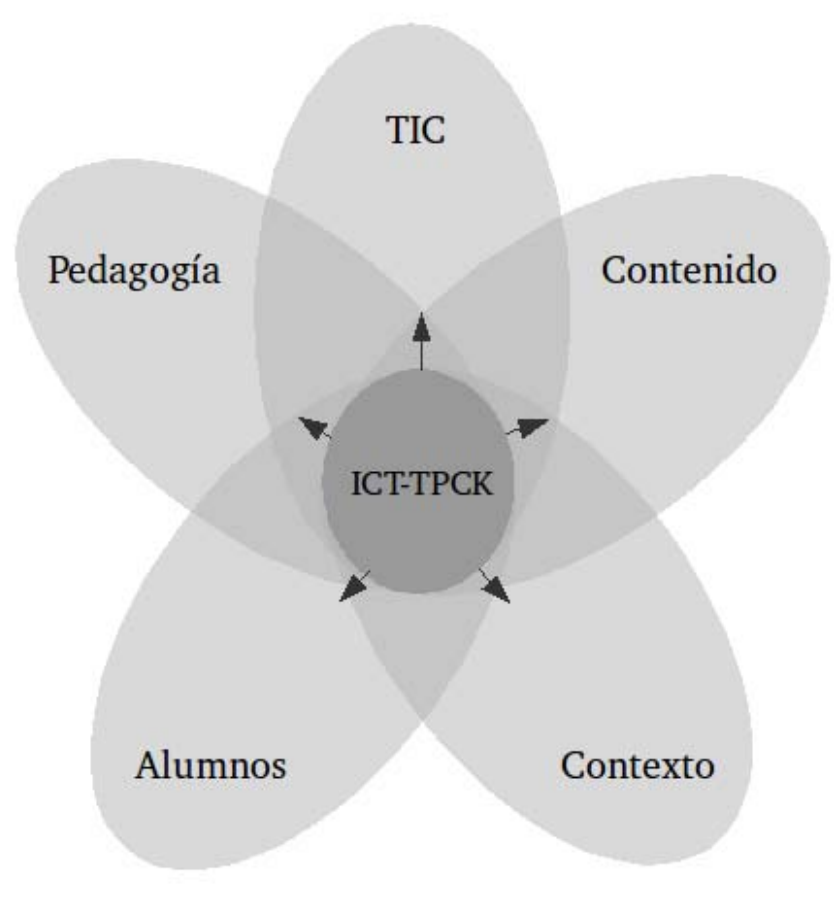

Fig. 5. El Modelo ICT-TPCK de Angeli y Valanides $(2009,159)$

ICT-TPCK se conceptualiza como una rama del TPCK, y éste sirve como base conceptual inicial para el modelo teórico. Así, los conocimientos base que lo constituyen, como se muestra en la Fig. 5, incluyen los tres elementos del TPCK que son el conocimiento del contenido curricular, el conocimiento pedagógico y de

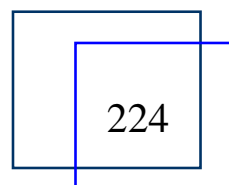

Jesús Valverde Berrocoso, María del Carmen Garrido Arroyo y Rosa Fernández Sánchez 


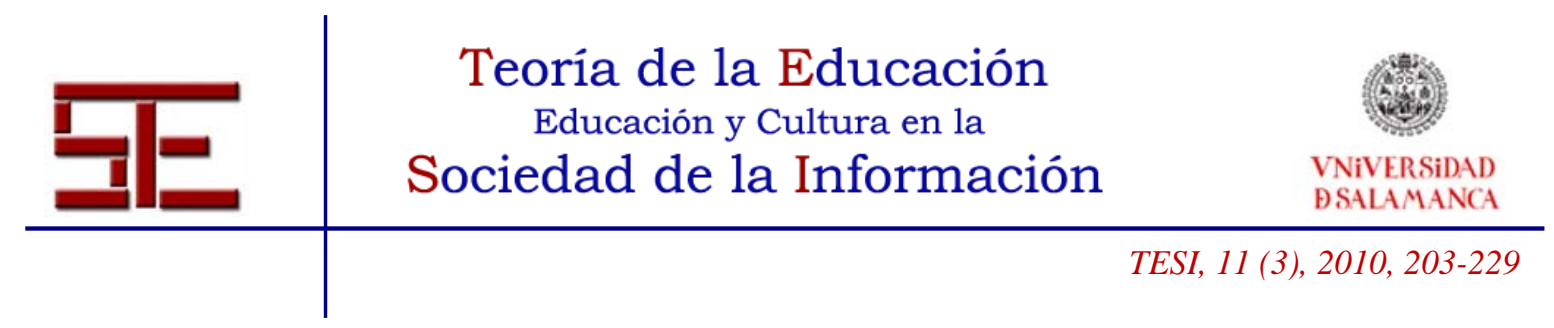

tecnología (restringido a las tecnologías de la información y la comunicación en este caso) y dos elementos adicionales: el conocimiento de los estudiantes y el conocimiento del contexto dentro del cual tiene lugar el aprendizaje.

Para Angeli \& Valadines (2009) cualquier preparación para el desarrollo del ICT-TPCK en la formación del profesorado debería tomar en consideración el corpus de conocimiento contextual y sumamente práctico del profesor. En otras palabras, el ICTTPCK no puede ser considerado como un corpus de conocimiento que exista independientemente de las creencias del profesor y de su experiencia práctica. Obviamente, cualquier enfoque que intente desarrollar el ICT-TPCK no debería sólo dar respuesta a las creencias y conocimentos del profesor, sino también debería estar centrado en el alumno. Puesto que el PCK es la base conceptual del TPCK, por colorario, PCK es también la columna vertebral y la base conceptual del ICT-TPCK, a pesar de la naturaleza especializada de éste. Esto quiere decir que el conocimiento del profesor de las representaciones de su materia y sus comprensiones de las concepciones de los alumnos y las dificultades relacionadas con el contenido también constituyen elementos clave del ICT-TPCK.

Por otra parte, el ICT-TPCK adopta ideas del constructivismo socio-cognitivo, porque considera que las transformaciones de contenido más eficaces son aquellas que crean conflicto cognitivo o socio-cognitivo y animan al diálogo y la negociación de significados entre estudiantes cuyas concepciones iniciales son diferentes (Valanides \& Angeli, 2008c). Cualquier transformación de la materia puede potencialmente tener diferentes efectos sobre los alumnos individuales debido a las diferencias en sus estilos cognitivos y de aprendizaje (Angeli \& Valanides, 2004; Valanides \& Angeli, 2006). De este modo, el ICT-TPCK es un modelo teórico que considera el aprendizaje de los estudiantes con diferentes estilos de aprendizaje, o diferentes formas de procesar la información, transformando el contenido con múltiples representaciones usando una variedad de medios tecnológicos de forma que alumnos y tecnología constituyen un sistema cognitivo conjunto.

\section{4.- CONCLUSIONES}

Ante la complejidad y dificultad que implica la integración de las tecnologías en las actividades de enseñanza y aprendizaje, la tecnología educativa necesita apoyar sus observaciones, análisis y propuestas en modelos teóricos que permitan a los investigadores alcanzar una comprensión profunda del proceso multifactorial y multivariado al que se enfrentan. El modelo TPCK aquí descrito, si bien no es un modelo novedoso, tiene la virtud de ofrecernos una estructura teórica que apoya tanto la investigación como la práctica. Se trata, además, de un modelo fundamentado en una amplia investigación empírica en la que, en los últimos años, están implicados un

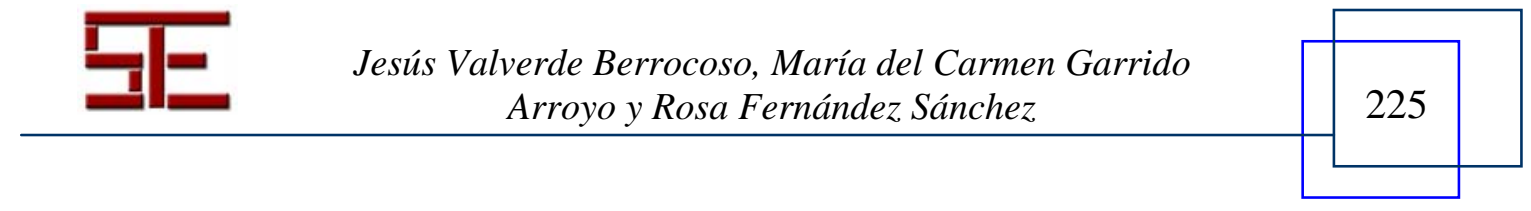




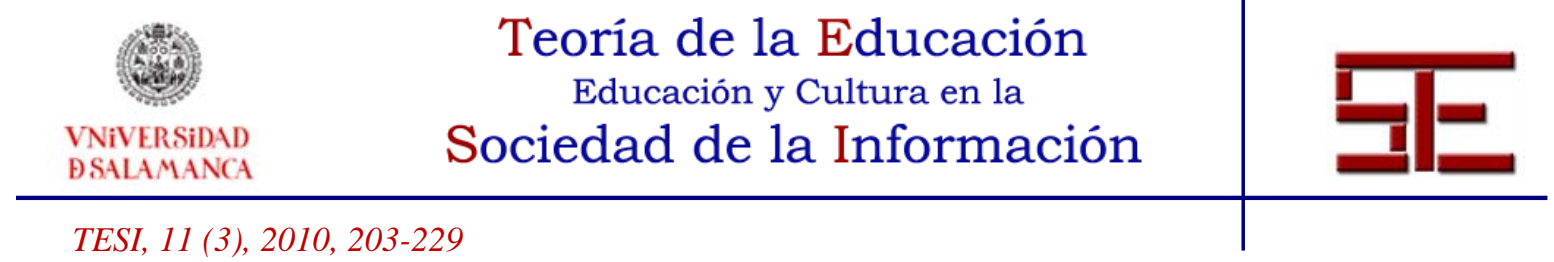

importante número de expertos. Es un modelo teórico que ha generado interés y debate entre los tecnólogos educativos y esto permite que sea revisado, actualizado y mejorado a medida que las evidencias corroboran o no sus hipótesis de partida. El TPCK es un tipo de conocimiento pragmático, muy ligado a la práctica docente $\mathrm{y}$, por consiguiente, con vocación de aplicar con inmediatez sus principios en la enseñanza con tecnologías. En este sentido, nos permite definir y valorar una buena práctica educativa con TIC. En definitiva, nos permite explorar y explicar fenómenos educativos ligados al uso de las TIC, encontrar nuevas preguntas para avanzar en la investigación educativa sobre tecnologías y diseñar programas formativos para el profesorado.

De la aplicación del modelo teórico TPCK a la formación del profesorado se puede concluir que las buenas prácticas educativas con TIC son acciones complejas y multidimensionales que exigen (1) comprender la representación y formulación de conceptos y procedimientos para su comprensión a través de las TIC; (2) desarrollar estrategias didácticas constructivistas que usen las TIC para la enseñanza de contenidos curriculares; (3) conocer las dificultades en el aprendizaje de conceptos y de qué forma las TIC pueden ayudar a superarlas, y (4) tomar en consideración el conocimiento previo de los alumnos, así como la epistemología del contenido curricular para comprender cómo las TIC pueden ser utilizadas para construir sobre el conocimiento pre-existente y desarrollar nuevas epistemologías. Estos conocimientos claramente van más allá del que posee aisladamente un experto en un contenido curricular (profesor con experiencia en la enseñanza de una disciplina), un experto en TIC (ingeniero informático) o un pedagogo experto (tecnólogo educativo), por lo que la formación de profesorado para la integración de las TIC exige un replanteamiento de los enfoques y las prácticas actuales excesivamente orientadas a la capacitación técnico-informática, sin relación con los contenidos curriculares específicos ni con los contextos de aplicación.

\section{5.- BIBLIOGRAFÍA}

Angeli, C. (2005). Transforming a teacher education method course through technology: Effects on preservice Teachers' technology competency. Computers \& Education, 45(4), 383-398.

Angeli, C. \& Valanides, N. (2004). Examining the effects of text-only and text-andvisual instructional materials on the achievement of field-dependent and fieldindependent learners during problem-solving with modeling software. Educational Technology Research and Development, 52 (4), 23-36.

- (2005). Preservice teachers as ICT designers: An instructional design model based on an expanded view of pedagogical content knowledge. Journal of Computer-Assisted Learning, 21 (4), 292-302.

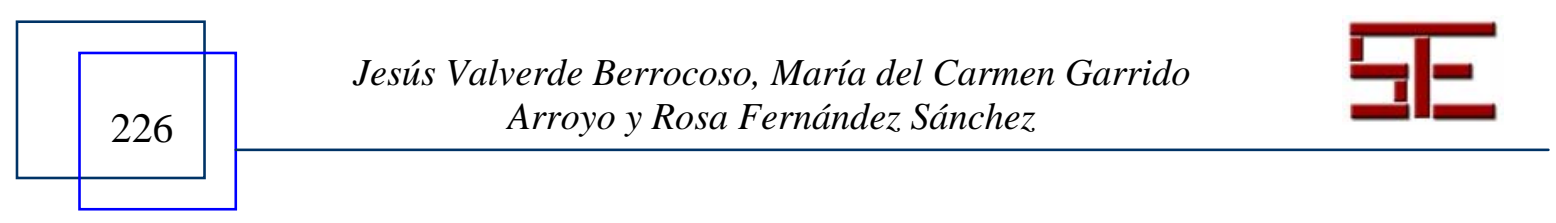




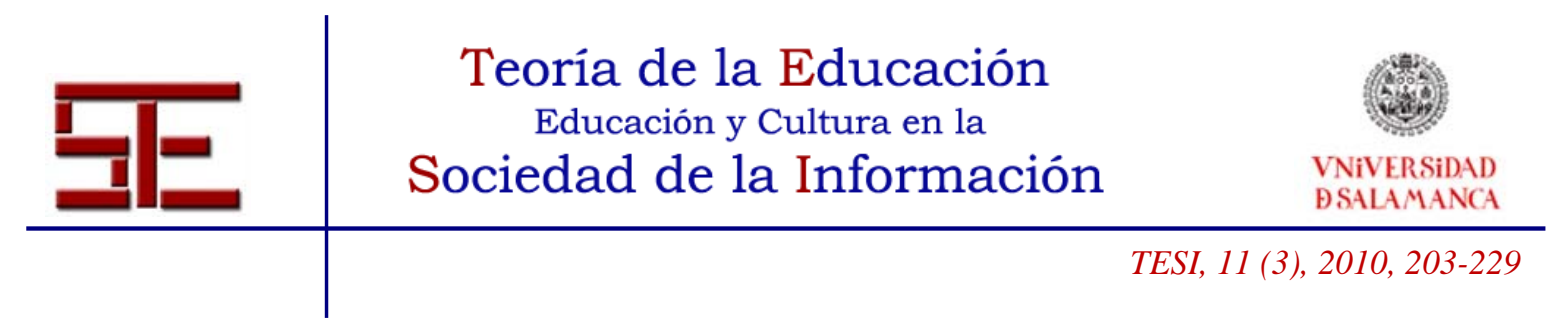

(2009). Epistemological and methodological issues for the conceptualization, development, and assessment of ICT-TPCK: Advances in technological pedagogical content knowledge (TPCK). Computers \& Education, 52, 154-168.

Area, M. (2004). Los medios y las tecnologías en la educación. Madrid: Pirámide.

Casanova Correa, J. (2007). Desafíos a la formación inicial del profesorado: buenas prácticas educativas en el contexto de la innovación con TIC. Revista Latinoamericana de Tecnología Educativa, 6 (2), 109-125. Disponible en http://campusvirtual.unex.es/cala/editio/.

Cochran, K. F., Derutier, J. A. \& King, R. A. (1993). Pedagogical content knowing: An integrative model for teacher preparation. Journal of Teacher Education, 44, 263-272.

Correa Gorospe, J. M. y Blanco Arbe, J. M. (2004). El proyecto Eskolaberri: evaluación de una experiencia de formación de directivos escolares para la integración de las nuevas tecnologías en centros de educación primaria. Revista Latinoamericana de Tecnología Educativa, 3 (1), 467-480. Disponible en http://campusvirtual.unex.es/cala/editio/.

De Pablos Pons, J. y Jiménez Cortés, R. (2007). Buenas prácticas con TIC apoyadas en las Políticas Educativas: claves conceptuales y derivaciones para la formación en competencias ECTS. Revista Latinoamericana de Tecnología Educativa, 6 (2), 15-28. Disponible en http://campusvirtual.unex.es/cala/editio/.

De Pablos, J., González, T. y González, A. (2008). El bienestar emocional del profesorado en los centros TIC como factor de innovación educativa. Revista Latinoamericana de Tecnología Educativa, 7 (2), 45-55. Disponible en http://campusvirtual.unex.es/cala/editio/.

Dewey, J. (2002). Cómo pensamos: nueva exposición de la relación entre pensamiento reflexivo y proceso educativo. Barcelona: Paidós.

Ertmer, P. A. (2005). Teacher pedagogical beliefs: The final frontier in our quest for technology integration. Educational Technology, Research and Development, 53 (4), 25-39.

Gibson, J. J. (1977). The Theory of Affordances. En RE. Shaw \& J. Bransford, Perceiving, Acting, and Knowing. Hillsdale, NJ: Lawrence Earlbaum Associates.

Kelly, A. E. (2003). Special issue on the role of design in educational research. Educational Researcher, 32 (1), 5-8.

Koehler, M. J. \& Mishra, P. (2008). Introducing TPCK. En AACTE (ed). Handbook of Technological Pedagogical Content Knowledge (TPCK) for Educators. New York: Routledge, (pp.3-30).

Koehler, M. J., Mishra, P. \& Yahya, K. (2007). Tracing the development of teacher knowledge in a design seminar: Integrating content, pedagogy, and technology. Computers \& Education, 49, 740-762.

Margerum-Lays, J. \& Marx, R. W. (2003). Teacher knowledge of educational technology: A case study of student/mentor teacher pairs. En Y. Zhao (ed.),

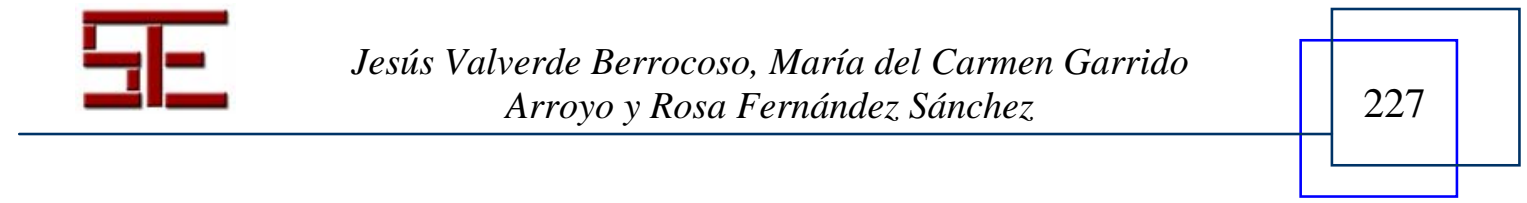




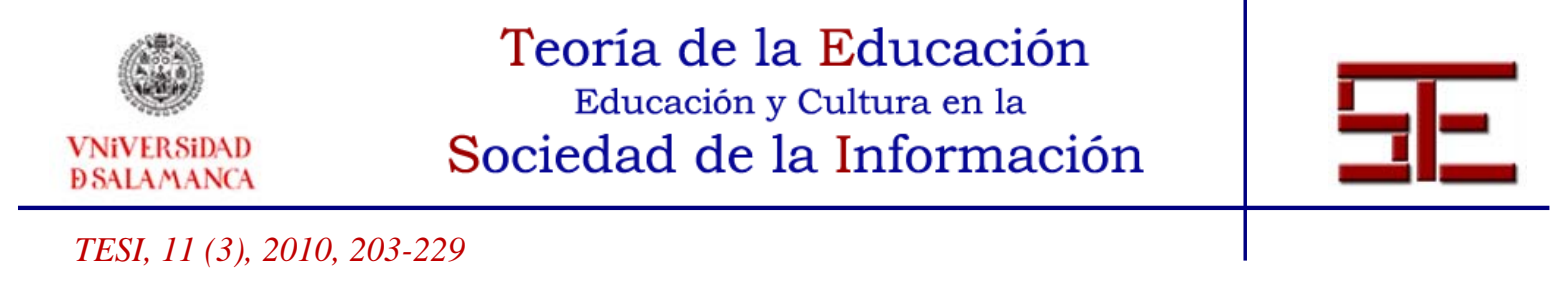

What should teachers know about technology? Perspectives and practices. Greenwich, CT: Information Age Publishing, (pp.123-159).

Mishra, P. \& Koehler, M. J. (2006). Technological pedagogical content knowledge: A new framework for teacher knowledge. Teachers College Record, 108 (6), 10171054.

National Research Council (1999). Being Fluent with Information Technology. Washington, D.C.: National Academic Press.

Niess, M. L. (2005). Preparing teachers to teach science and mathematics with technology: Developing a technology pedagogical content knowledge. Teaching and Teacher Education, 21(5), 509-523.

- (2008). Guiding preservice teachers in TPCK. En AACTE Committee on Innovation and Technology (ed.), Handbook of Technological Pedagogical Content Knowledge (TPCK) for Educators. New York: Routledge, (pp.223-250).

Perkins, D. N. (1986). Knowledge as design. Hillsdale, NJ: Lawrence Earlbaum Associates.

Pierson, M. E. (2001). Technology integration practice as a function of pedagogical expertise. Journal of Research on Computing in Education, 33 (4), 413-429.

Prensky, M. (2001). Digital natives, digital immigrants. On the Horizon, 9(5), 1-2. Disponible en: http://www.marcprensky.com/writing/Prensky\%20\%20Digital\%20Natives,\%20Digital\%20Immigrants\%20-\%20Part1.pdf .

- $\quad$ (2006). Listen to the natives, Educational Leadership. 63 (4), 8-13.

Raposo, M., Fuentes, E. y González, M. (2006). Desarrollo de competencias tecnológicas en la formación inicial de maestros. Revista Latinoamericana de Tecnología Educativa, 5 (2), 525-537. Disponible en http://campusvirtual.unex.es/cala/editio/.

Rittel, H. W. \& Webber, M. M. (1973). Dilemmas in a General Theory of Planning. Policy Sciences 4, 155-169. Disponible en: http://www.uctc.net/mwebber/Rittel+Webber+Dilemmas+General_Theory_of_P lanning.pdf.

Schmidt, D. A. \& Gurbo, M. (2008). TPCK in K-6 literacy education. En AACTE Committee on Innovation and Technology (ed.), Handbook of Technological Pedagogical Content Knowledge (TPCK) for Educators, New York: Routledge. (pp61-85).

Schön, D.A. (1998). El profesional reflexivo: cómo piensan los profesionales cuando actúan. Barcelona: Paidós.

(2008). La formación de profesionales reflexivos: hacia un nuevo diseño de la enseñanza y el aprendizaje en las profesiones. Barcelona: Paidós.

Shulman, L. S. (1986). Those who understand: Knowledge growth in teaching. Educational Researcher, 15(2), 4-14.

- (1987). Knowledge and Teaching: Foundations of the New Reform. Harvard Educational Review, 57 (1), 1-2. Trad. cast. en: Shulman, L.S. (2005).

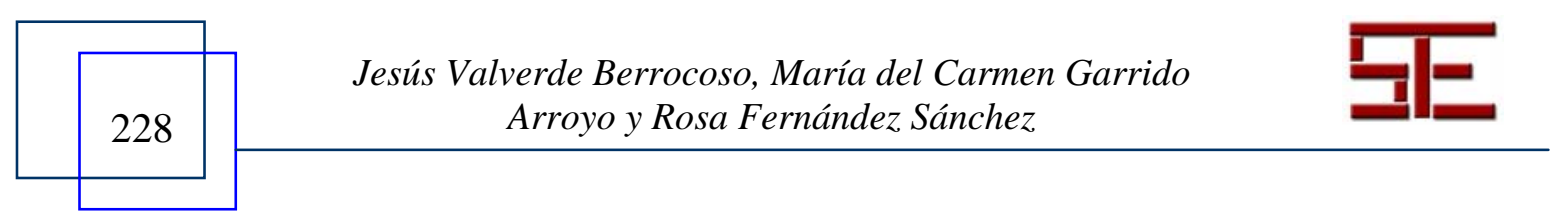




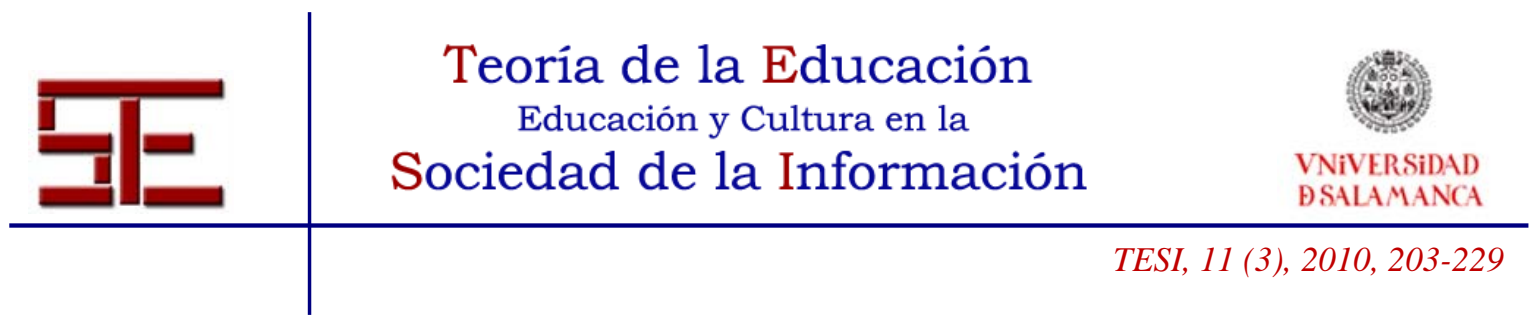

Conocimiento y Enseñanza: fundamentos de una nueva reforma. Profesorado. Revista de currículum y formación del profesorado, 9, 2 [http://www.ugr.es/local/recfpro/Rev92ART1.pdf ].

Valanides, N. \& Angeli, C. (2006). Preparing preservice elementary teachers to teach science through computer models. Contemporary Issues in Technology and Teacher Education - Science, 6(1), 87-98.

- (2008a). Learning and teaching about scientific models with a computer modeling tool. Computers in Human Behavior, 24, 220-233.

- (2008b). Professional development for computer-enhanced learning: A case study with science teachers. Research in Science and Technological Education, 26(1), 3-12.

- (2008c). Distributed cognition in a sixth-grade classroom: An attempt to overcome alternative conceptions about light and color. Journal of Research on Technology in Education, 40(3), 309-336.

Valverde Berrocoso, J., Barroso Osuna, J., Garrido Arroyo, Ma del C. y Ameijeras Saiz, R. (1999). Los principios pedagógicos constructivistas en la formación inicial del profesorado y las nuevas tecnologías. En J. Argos y $\mathrm{M}^{\mathrm{a}} \mathrm{P}$. Esquerra, Principios del Curriculum. Santander: Servicio de Publicaciones de la Universidad de Cantabria, (pp.325-335).

Valverde Berrocoso, J. (2002). Formación del Profesorado para el uso educativo de las Tecnologías de la Información y la Comunicación. Revista Latinoamericana de Tecnología Educativa, 1 (2), 9-28. Disponible en http://campusvirtual.unex.es/cala/editio/.

Zhao, Y. (2003). What teachers should know about technology: Perspectives and practices. Greenwich, CT: Information Age Publishing.

Zhao, Y., Pugh, K., Sheldon, S. \& Byers, J. L. (2002). Conditions for classroom technology innovations. Teachers College Record, 104 (3), 482-515.

Para citar el presente artículo puede utilizar la siguiente referencia:

Valverde Berrocoso, J., Garrido Arroyo, Mª C. y Fernández Sánchez, R..: (2010). "Enseñar y aprender con tecnologías: un modelo teórico para las buenas prácticas educativas con TIC”. En De Pablos Pons, J. (Coord.) Buenas prácticas de enseñanza con TIC [monográfico en línea]. Revista Electrónica Teoría de la Educación: Educación y Cultura en la Sociedad de la Información. Vol. 11, $\mathrm{n}^{0} 1$. Universidad de Salamanca, pp. 203-229. [Fecha de consulta: dd/mm/aaaa].

http://revistatesi.usal.es/ revistas_trabajo/index.php/revistatesi/article/view/5840/5866 ISSN: 1138-9737

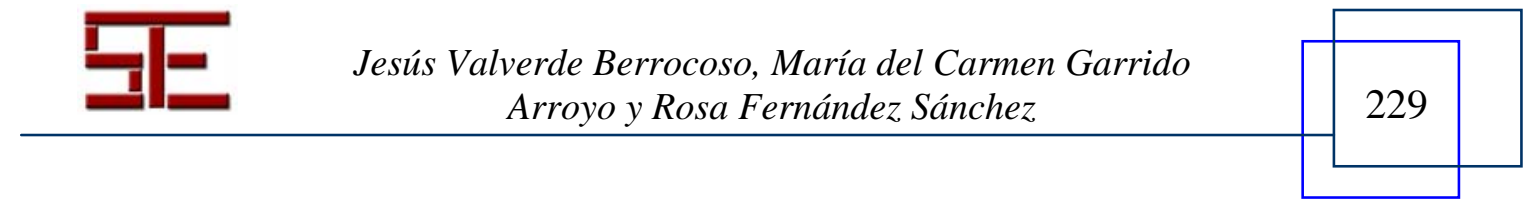

